\title{
Space and Time Evolution of the Electrostatic Potential of a Visual Pigment
}

\author{
Federico Melaccio $^{\dagger,{ }^{*}}$, Nicolas Calimet ${ }^{\dagger \dagger}$, Igor Schapiro ${ }^{\ddagger}$, Alessio Valentini ${ }^{\dagger}$, Marco Cecchini $^{\dagger \dagger}$, \\ and Massimo Olivucci ${ }^{+, \boldsymbol{\top}, \#^{*}}$.
}

ๆศ Dipartimento di Biotecnologie, Chimica e Farmacia, Università di Siena, via A. Moro 2, I-5310o Siena, Italy, ${ }^{\dagger}$ Department of Chemistry, Bowling Green State University, Bowling Green, OH 43403, USA, ${ }^{\dagger \dagger}$ ISIS, UMR 7006 CNRS, Université de Strasbourg, F-67083 Strasbourg Cedex, France, ${ }^{\ddagger}$ Fritz Haber Center for Molecular Dynamics, Institute of Chemistry, The Hebrew University of Jerusalem, Jerusalem 91904, Israel, " Institut de Physique et Chimie des Matériaux de Strasbourg, UMR 7504 Université de Strasbourg_CNRS, F-67034 Strasbourg, France.

\section{Supporting Material}

\author{
So. Experimental Section
}

S1. Excited State Trajectory of Bovine Rhodopsin

S2. Semi-classical trajectories of a reduced $\mathrm{Rh}$ model

S3. Electrostatic Potential (ESP) Calculations

S4. $\mathbf{p K}_{\mathrm{a}}$ Calculations 


\section{So. Experimental Section}

\section{So.1 Trajectory calculations}

All semi-classical trajectories are computed at the CASPT2//CASSCF/6-31 $\mathrm{G}^{*}$ level of theory as for the Rh trajectory which is from ref. 1 . The gas-phase equilibrium $4 \mathrm{MePSB} 3$ structure is 25 degrees pre-twisted to mimic the distortion imposed by the protein cavity in the natural system. The 100 initial conditions (structures and velocities) are generated according to a simulated Boltzmann distribution at $298 \mathrm{~K}$. The surface hopping events (i.e., non-adiabatic transitions) were imposed using Tully's fewest switches algorithm. Full details are provided in sections 1 and 2 of the SI.

\section{So.2 Electrostatic Potential}

The Poisson-Boltzmann continuum electrostatic equation was solved for each of the 176 trajectory snapshots of the Rh trajectory (see full details in SI section 3). Briefly, to compute the EPS change on each residue we used Mulliken atom charges for PSB11. The ESPs computed on a 3D grid within 1.5 $\AA$ of each residue (side chain) atom were averaged to give $\mathrm{Vn}$, the ESP of the residue at the n-th snapshot, and the Vn-Vo variation (Vo is the initial So value). For each selected residue, $\mathrm{Vn}$ was computed in presence of all protein charges (from the AMBER94 force field ${ }^{2}$ ) except those of the selected residue itself, which were set to zero. A similar procedure was used to visualize the EPS on the chromophore cavity surface. The color scale was set from minus 100 to 100 (in units of kT/e) All the 176 images were merged into a movie file which is part of the SI.

\section{So.3 $p K_{a}$}

For each ionizable residue the protonation state at $\mathrm{pH} 7$ was predicted based on continuum electrostatics and the multiple-site titration approach ${ }^{3}$; see full details in SI section 4. Using this approach, titration profiles were computed over a wide $\mathrm{pH}$ range, i.e. from -14 to +28 , to provide a $\mathrm{pKa}$ estimate even for very acidic or very basic residues; typical titration curves are computed from o to $+14 \cdot{ }^{4}$ All other coordinates and charges were left identical. Solvent-exposed ionizable residues that are far from the retinal-binding pocket in the crystal structure of Rh were assigned a fixed neutral state following previous work by $\mathrm{us}^{1,5}$. His195, which is located at the extracellular end of the membrane but changes its predicted protonation upon neutralization of the solvent-exposed residues, was considered to be ionizable.

\section{S1. Excited State Trajectory of Bovine Rhodopsin}

The quantum mechanics / molecular mechanics (QM/MM) excited state trajectory of bovine rhodopsin (Rh) used for our analysis was taken from ref. 1, where the computational setup is given in detail. Here we provide a summary of relevant information. The QM/MM frontier runs across the $C \delta-C \varepsilon$ bond of the retinal bound Lys296 and a hydrogen link atom is used to cap the pending valence ${ }^{6}$ (Figure $\mathrm{S}_{1}$ ). The QM region is treated using the $a b$ initio multiconfigurational Complete Active Space / Self Consistent Field (CASSCF) ${ }^{7}$ method, which partly account for electron correlation at an affordable computational cost, thus giving the required accuracy to study photochemical reactivity. The active space includes the full retinal $\pi$-system, which corresponds to 12 electrons in 12 orbitals. The AMBER force field ${ }^{2}$ is used for the MM region. The active space includes the full retinal $\pi$-system, which corresponds to 12 electrons in 12 orbitals. The AMBER force field\{Cornell 1995 \} is used for the MM region. In principle, a computational setup including: (i) a more extended QM subsystem comprising the cavity residues or (ii) a polarizable force field is desirable. However, its realization appears presently difficult due to the difficulty in selecting a significant "cavity subset" small enough to be incorporated in the QM subsystem for point $\mathrm{i}$ and, the need of preliminary studies validating the currently available force fields for point ii.

The protein is kept frozen along the time evolution, except for the sidechain of Lys296, since the process is fast enough to assume little thermal motion of the chromophore surroundings. CASSCF energies and snapshot times are corrected by scaling them to reproduce the more accurate Complete Active Space Perturbation Theory of order 2 (CASPT2) ${ }^{8}$ energy profile, in order to fully recover electron correlation, following the procedure in ref. ${ }^{9}$. The trajectory was released from the Franck-Condon (FC) point on S1 with zero initial velocities to approximate the centroid of multiple trajectories with 
thermally sampled initial velocities, which works well for short excited state lifetimes $(<100 \mathrm{fs})$. Our Rh trajectory is propagated according to the velocity Verlet method by using the QM/MM force and a time step of $1 \mathrm{fs}$. At each snapshot the So-S1 energy gap and the wavefunction character are used to assess whether a state degeneracy (conical intersection, $\mathrm{CI}$ ) is reached. If so, the energy and gradient are then evaluated on So. The degeneracy is reached at 94 fs (unscaled time) and the overall trajectory consists of $176 \mathrm{fs}$ (Figure S2). Molcas 7.5 development version ${ }^{10}$ and Tinker $4.2^{11}$ were used. In Figure $\mathrm{S}_{3}$ we report the characterization of a CASSCF trajectory analogue to that of Figure 1 in the main text and Figure S2 below but featuring the side chains of the chromophore cavity free to relax. The chromophore cavity is defined by the set of side chains of the protein ground state equilibrium structure characterized by having at least one atom located within 4 Å from any atom of the retinal chromophore.

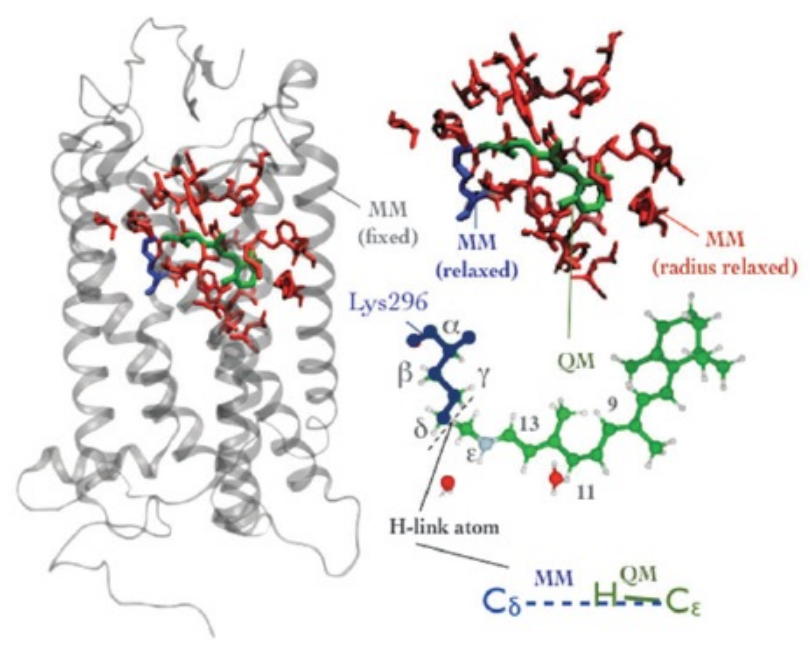

Figure S1. QM/MM partition of Rh. Taken from ref. ${ }^{12}$.

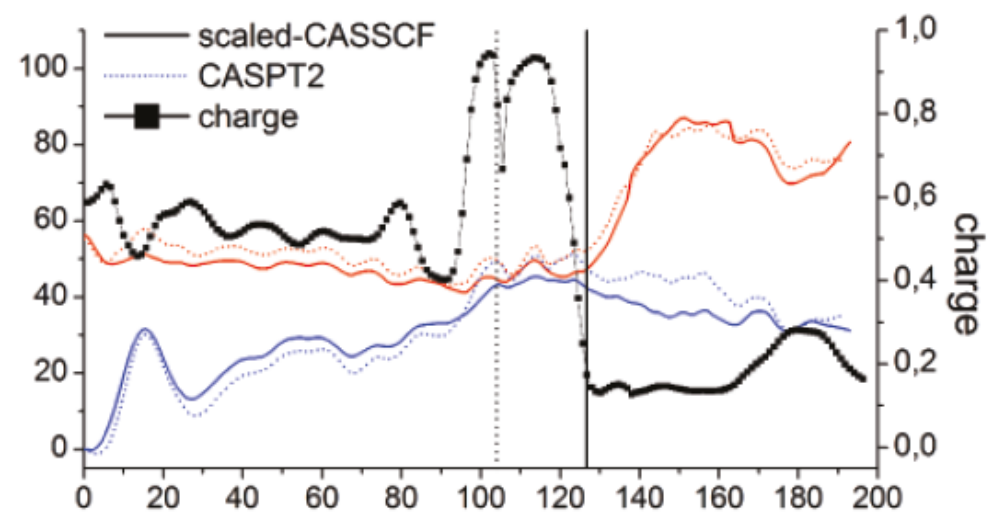

Figure $\mathrm{S}_{2} . \mathrm{S}_{1}$ and $\mathrm{S}_{\mathrm{o}}$ energies and charge vs time for Rh excited state dynamics (adapted from ref. 1)

In order to assess the sensitivity to a flexible chromophore cavity environment, we have compute an additional excited state trajectory. In this trajectory the sidechains which have at least one atom within 4 A distance to the chromophore were flexible. That means they were propagated in time with the chromophore. The results are shown in Fig. S3. It was found that this setup does not change the documented ESP evolution of the cavity shape during the ca. 200 fs photoisomerization time. 

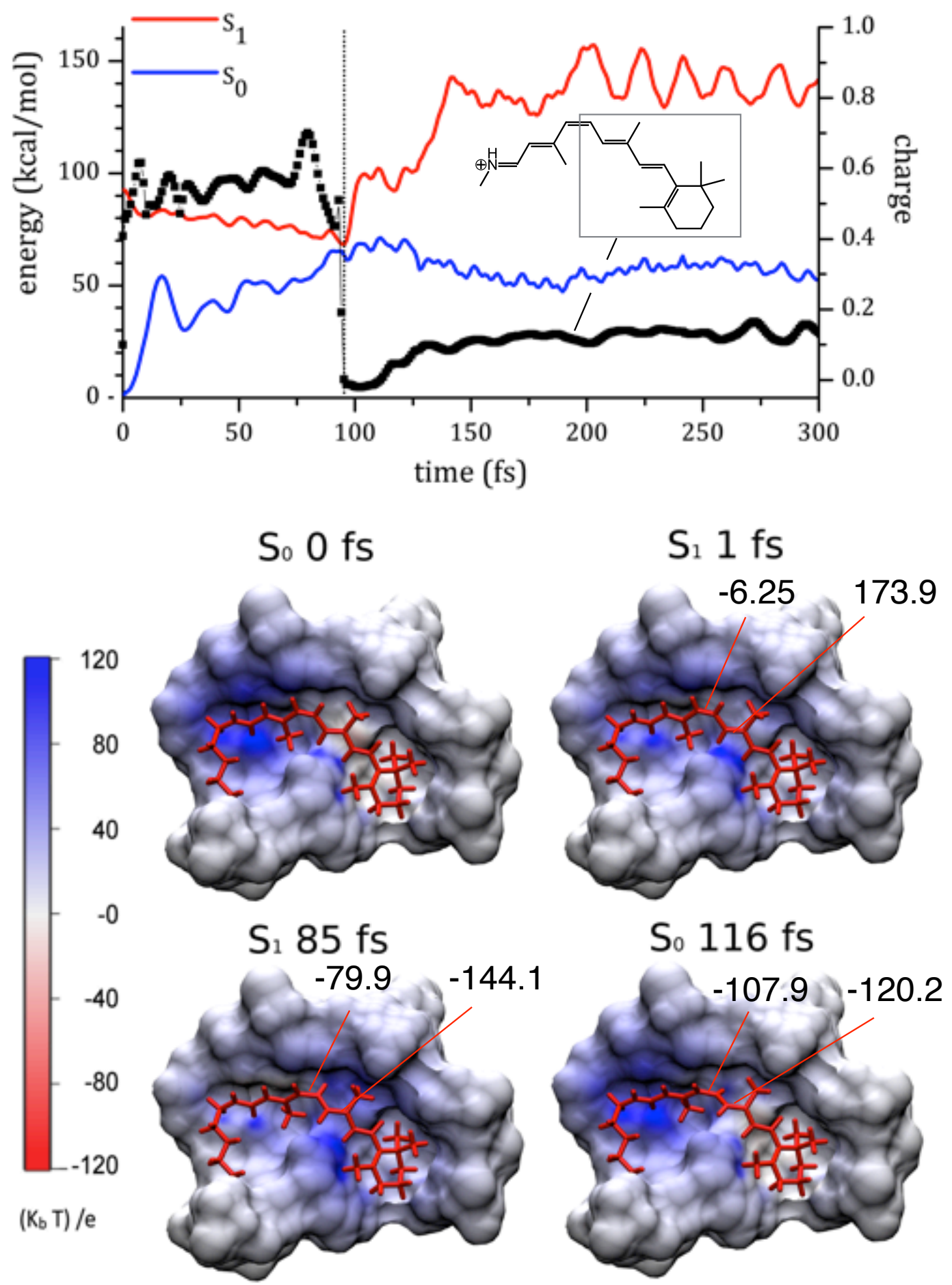

Figure S3. Top. Evolution of the charge distribution along the 300 fs trajectory computed using the same methodology reported in ref. 1 and in section So.2 but featuring chromophore cavity residues (all residues with at least one atom at a 4 $\AA$ Aistance or less from any atom of the chromophore) free to relax. The plotted charge corresponds to the total Mulliken charge of the framed PSB11 region. Bottom. Trajectory snapshots documenting the ESP change on the chromophore cavity of bovine Rh. The visualized cavity includes residues Ser186, Glu181, Tyr191, Tyr192 and Tyr268 of Figure 3A. Positive potential values are given in blue. The values of the $\mathrm{C}_{11}-\mathrm{C}_{12}$ and $\mathrm{C}_{9}-\mathrm{C}_{10}$ dihedral angles (in degrees) describing the aborted bicycle-pedal photoisomerization ${ }^{1}$ coordinates are also given. The snapshots have to be compared with the ones in Fig. 2. All images were merged into a movie file (Supplementary Video)

\section{S2. Semi-classical trajectories of a reduced $\mathrm{Rh}$ model}


In order to show that our reference Rh Franck-Condon trajectory has the average features of a reactive trajectory representing the average behavior of a population, we computed a series of surface hopping molecular dynamics trajectories for the reduced model system 4-methyl-penta-2,4-dieniminium cation (4MePSB3). A set of 100 thermally sampled initial conditions was prepared according to the Boltzmann distribution at $298 \mathrm{~K}$ and at the HF level of theory. The initial conditions were generated by quasi-classical fixed local mode sampling at $298 \mathrm{~K}$ and 1 atm, as implemented in Gaussian $^{13}$ (and based on VENUS96 ${ }^{14}$ ), and used to start trajectory calculations following the same approach as the reference trajectory (Section S1), except for the electronic CASSCF wavefunction featuring electrons in 6 orbitals (e.g. the complete $\pi$-system of ${ }_{4} \mathrm{MePSB}_{3}$ ). The surface hopping events (i.e., non-adiabatic transitions) were imposed using Tully's fewest switches algorithm. ${ }^{15}$ Full details about the technical setup are given in ref. 16 Briefly, the time-dependent wavefunction and the population of each electronic state in time were determined by performing 200 substeps of integrations $\left(5 \cdot 10^{-3} \mathrm{fs}\right)$ of the time-dependent Schrödinger equation for each Newton equation of motion integration step ( $1 \mathrm{fs}$ ). The projection of the derivative coupling vector along the trajectory was computed by finite differences at each step of the nuclear motion integration. In order to determine the derivative coupling elements for each electronic-integration substep, an interpolation-extrapolation scheme was used. ${ }^{17}$ Finally, the decoherence correction due to Granucci and Persico was employed to improve the internal consistency of the surface hopping algorithm. ${ }^{18}$

\section{S3. Electrostatic Potential (ESP) Calculations}

\section{S3.1 Visualization of Retinal ESP on the protein cavity surface (Main text Figure 1)}

The visualization of the retinal ESP projected onto the protein cavity made use of custom Linux bash scripts, interfacing to the molecular viewer program VMD (Visual Molecular Dynamics) ${ }^{19}$ and the APBS (Adaptive Poisson-Boltzmann Solver $)^{20}$ program for the calculation of electrostatic potential. The chromophore geometries along the trajectory were used from the Molcas ${ }^{10}$ output (concatenated standard XYZ files), while the (frozen) protein geometry was available in the Tinker $^{11}$ XYZ format. Our scripts generated 176 snapshots in the Tinker XYZ format by replacing the chromophore initial coordinates with those from each trajectory snapshots. Those files were subsequently converted to PDB (Tinker utility pdbxyz) and then to the PQR format (PDB2PQR utility ${ }^{21}$ ). PQR files are re-formatted PDB to include partial charges in atomic units and van der Waals radii in $\AA$, for numerically solving the Poisson-Boltzmann continuum electrostatic equation by APBS. We used Mulliken atom charges for retinal, while all protein charges were set to zero to isolate the chromophore effect. Van der Waals radii were taken from the AMBER ${ }^{2}$ force field. The APBS calculation on the 176 PQR files generated a three dimensional grid of electrostatic potential values in units of kT/e, in the DX format with the default value of 161 points in each dimension (about $0.5 \AA$ spacing). Subsequently, a custom VMD Tcl script was used to load each PDB snapshot file and the associated DX grid, which can be visualized by VMD providing a color scale matching the order of magnitude of electrostatic potential values. More specifically, the color scale was set from minus 100 to 100 (in units of kT/e) and the DX grid was plotted onto the default Surf representation (1.4 $\AA$ probe). The Tcl script looped over the 176 snapshots to render the colored surface as described above, and to export a bitmap image file for each snapshot, including the four images in Figure 1 B. All the 176 images were merged into a movie file (Supplementary Video).

\section{S3.2 Quantitative evaluation of ESP on single cavity residues (Main text Figure 3)}

The quantitative assessment of ESP variation over the cavity space was performed by using a modification of the aforementioned procedure in Section S3.1 to account for the global protein effect and isolate the ESP acting on single residues. More specifically:

$\checkmark$ All protein atoms were assigned the AMBER partial charges.

$\checkmark$ Lys296 sidechain got the QM/MM re-parametrized charges as in ref. 22.

$\checkmark$ To guarantee an integer zero total charge, the (small) link atom Mulliken charge was assigned to the Lys296 C $\varepsilon$ replacing its zero charge from the QM/MM re-parametrization.

Each residue was assigned zero atomic charges, then the same file conversions and APBS calculations were run to generate ESP DX grids, where the protein and chromophore effect on the single residue could be assessed. 9 cavity polar or charged residues and 2 water molecules were selected, as reported in the Main Text. A custom scripts was then used to evaluate electrostatic effects more quantitatively. The ESP values associated to DX grid points within $1.5 \AA ̊$ of each zero 
charge residue were selected and averaged to give the ESP value at the $n$-th snapshot, $V_{n}$, with $V_{o}$ being the initial value for the So ground state (Table $S_{1}$ ). Then the time evolution of the $V_{n}-V_{o}$ difference was obtained as a measure of the ESP variation along the trajectory with respect to initial values, to generate the Figure ${ }_{3} \mathrm{~B}$ plot in the Main Text. Table S2 reports the corresponding data.

Table S1. Absolute ESP values along time for each zero charged residue. Values rounded at 3 decimal digits.

\begin{tabular}{|c|c|c|c|c|c|c|c|c|c|c|c|}
\hline Time & Glu113 & Glu122 $^{\mathrm{H}}$ & Ser186 & Thr94 & Thrus & Tyri91 & Tyr268 & Watı & Wat2 & Glu181 ${ }^{H}$ & Tyrig2 \\
\hline o & $45 \cdot 997$ & -3.587 & 8.861 & 1.165 & -1.343 & -3.081 & 1.714 & 19.334 & -2.343 & 14.841 & 0.390 \\
\hline 1 & 38.007 & 1.094 & -0.761 & -6.344 & 5.959 & -0.572 & 5.151 & 12.200 & -11.749 & 14.235 & 0.633 \\
\hline 2 & 37.913 & 1.114 & -0.765 & -6.399 & 5.969 & -0.565 & 5.165 & 12.213 & -11.826 & 14.234 & 0.634 \\
\hline 3 & 37.701 & 1.184 & -0.794 & -6.557 & $5 \cdot 990$ & $-0.55^{2}$ & 5.186 & 12.197 & -11.979 & 14.208 & 0.631 \\
\hline 4 & $37 \cdot 386$ & 1.297 & -0.821 & -6.823 & 6.030 & -0.533 & 5.209 & 12.133 & -12.229 & 14.148 & 0.622 \\
\hline 5 & 37.093 & 1.476 & -0.927 & -7.145 & 6.103 & -0.500 & 5.234 & 11.990 & -12.462 & 14.064 & 0.610 \\
\hline 6 & 37.014 & 1.627 & -0.929 & $-7 \cdot 360$ & 6.079 & -0.501 & 5.188 & 11.865 & -12.539 & 13.962 & 0.590 \\
\hline 7 & 37.438 & 1.580 & -0.620 & -7.209 & $5 \cdot 791$ & -0.601 & 4.998 & 11.946 & -12.138 & 13.861 & 0.557 \\
\hline 8 & 38.407 & 1.247 & 0.156 & -6.602 & 5.183 & -0.816 & 4.667 & 12.422 & -11.159 & 13.837 & 0.525 \\
\hline 9 & 39.539 & 0.780 & 1.115 & $-5.75^{8}$ & 4.442 & -1.084 & 4.293 & 13.061 & -10.001 & 13.844 & 0.493 \\
\hline 10 & 40.359 & 0.423 & 1.904 & -5.122 & 3.835 & -1.293 & 4.013 & $13 \cdot 515$ & -9.148 & 13.822 & 0.462 \\
\hline 11 & 40.805 & 0.201 & 2.487 & -4.692 & $3 \cdot 387$ & -1.442 & 3.823 & 13.810 & -8.611 & 13.794 & 0.437 \\
\hline 12 & 41.047 & 0.031 & 2.994 & -4.377 & 3.029 & -1.551 & 3.683 & 14.045 & -8.281 & 13.768 & 0.418 \\
\hline 13 & 41.101 & -0.068 & $3 \cdot 344$ & -4.183 & 2.793 & -1.617 & 3.610 & 14.146 & -8.160 & 13.723 & 0.401 \\
\hline 14 & 41.017 & -0.042 & 3.480 & -4.133 & 2.762 & -1.614 & 3.623 & 14.072 & -8.229 & 13.685 & 0.396 \\
\hline 15 & 40.770 & 0.079 & 3.336 & -4.232 & 2.915 & -1.544 & $3 \cdot 717$ & 13.749 & -8.526 & 13.626 & 0.395 \\
\hline 16 & 40.476 & 0.286 & 3.022 & -4.410 & 3.212 & -1.435 & 3.858 & 13.227 & -8.931 & 13.534 & 0.394 \\
\hline 17 & 40.201 & 0.468 & 2.663 & -4.578 & 3.527 & -1.322 & 4.001 & 12.689 & -9.335 & $13.45^{8}$ & 0.398 \\
\hline 18 & 40.004 & 0.570 & 2.416 & -4.617 & 3.750 & -1.244 & 4.088 & 12.226 & -9.604 & $13 \cdot 391$ & 0.398 \\
\hline 19 & 39.968 & 0.564 & 2.347 & $-4 \cdot 512$ & 3.834 & -1.213 & 4.126 & 12.008 & -9.665 & 13.363 & 0.399 \\
\hline 20 & 39.860 & 0.521 & 2.403 & -4.443 & 3.866 & -1.210 & 4.125 & 11.911 & -9.720 & 13.348 & 0.399 \\
\hline 21 & 39.672 & 0.518 & 2.393 & -4.485 & 3.901 & -1.198 & 4.132 & 11.848 & -9.859 & 13.324 & 0.400 \\
\hline 22 & $39 \cdot 344$ & 0.618 & 2.253 & -4.716 & 4.042 & -1.153 & 4.188 & 11.755 & -10.164 & 13.300 & 0.403 \\
\hline 23 & 38.979 & 0.788 & 2.037 & -5.096 & 4.235 & -1.092 & 4.257 & 11.579 & -10.558 & 13.253 & 0.405 \\
\hline 24 & 38.825 & 0.938 & 1.834 & -5.426 & $4 \cdot 385$ & -1.047 & 4.305 & 11.481 & -10.852 & 13.212 & 0.405 \\
\hline 25 & 38.861 & 1.050 & 1.747 & -5.669 & 4.467 & -1.038 & 4.308 & 11.474 & -10.982 & 13.176 & 0.402 \\
\hline 26 & 39.015 & 1.089 & 1.788 & -5.789 & 4.456 & -1.056 & 4.273 & 11.564 & -11.024 & 13.165 & 0.400 \\
\hline 27 & 39.220 & 1.085 & 1.918 & -5.806 & $4 \cdot 394$ & -1.098 & 4.199 & 11.722 & -11.006 & 13.170 & 0.397 \\
\hline 28 & $39 \cdot 374$ & 1.040 & 2.132 & $-5 \cdot 744$ & 4.258 & -1.165 & 4.097 & 11.894 & -10.979 & 13.161 & 0.387 \\
\hline 29 & 39.488 & 0.986 & 2.421 & -5.611 & 4.127 & -1.238 & 3.983 & 12.125 & -10.947 & 13.178 & 0.382 \\
\hline 30 & 39.533 & 0.902 & 2.702 & -5.438 & 3.960 & -1.314 & 3.866 & 12.334 & -10.940 & 13.195 & 0.375 \\
\hline 31 & 39.591 & 0.825 & 2.975 & -5.212 & 3.830 & -1.382 & 3.764 & 12.532 & -10.929 & 13.219 & 0.370 \\
\hline 32 & 39.667 & 0.765 & 3.148 & -4.992 & 3.726 & -1.432 & 3.677 & 12.662 & -10.915 & 13.247 & 0.367 \\
\hline 33 & 39.798 & 0.710 & 3.239 & $-4 \cdot 771$ & 3.658 & -1.476 & 3.611 & 12.747 & -10.882 & 13.263 & 0.363 \\
\hline 34 & 39.907 & 0.696 & 3.212 & -4.617 & 3.632 & -1.493 & $3 \cdot 571$ & 12.727 & -10.885 & 13.283 & 0.361 \\
\hline 35 & 40.006 & 0.717 & 3.094 & -4.503 & 3.669 & -1.488 & 3.557 & 12.642 & -10.895 & 13.294 & 0.362 \\
\hline 36 & 39.987 & 0.754 & 2.888 & -4.468 & 3.708 & -1.480 & 3.556 & 12.479 & -10.944 & 13.288 & 0.357 \\
\hline 37 & 39.888 & 0.801 & 2.673 & -4.487 & 3.725 & -1.464 & $3 \cdot 555$ & 12.317 & -10.977 & 13.286 & 0.355 \\
\hline 38 & $39 \cdot 722$ & 0.852 & 2.460 & $-4 \cdot 581$ & 3.767 & -1.445 & 3.563 & 12.165 & -11.010 & 13.286 & 0.353 \\
\hline 39 & 39.545 & 0.873 & 2.279 & -4.668 & 3.760 & -1.434 & $3 \cdot 564$ & 12.079 & -10.966 & 13.290 & 0.352 \\
\hline 40 & 39.452 & 0.875 & 2.127 & -4.736 & 3.704 & -1.442 & 3.544 & 12.034 & -10.860 & 13.289 & 0.346 \\
\hline 41 & 39.476 & 0.850 & 1.999 & -4.767 & 3.631 & -1.449 & 3.525 & 11.985 & -10.677 & 13.299 & 0.342 \\
\hline 42 & 39.640 & 0.806 & 1.905 & -4.765 & $3 \cdot 524$ & -1.466 & 3.489 & 11.998 & -10.410 & 13.308 & 0.338 \\
\hline 43 & 39.893 & 0.765 & 1.840 & -4.756 & 3.424 & -1.478 & 3.461 & 12.065 & -10.098 & 13.335 & 0.339 \\
\hline 44 & 40.105 & 0.690 & 1.852 & -4.718 & 3.267 & -1.503 & 3.426 & 12.172 & -9.812 & $13 \cdot 371$ & 0.340 \\
\hline 45 & 40.290 & 0.606 & 1.913 & -4.666 & 3.106 & -1.529 & $3 \cdot 391$ & 12.343 & -9.538 & 13.421 & 0.344 \\
\hline 46 & 40.364 & 0.485 & 2.040 & -4.600 & 2.910 & -1.557 & 3.359 & 12.556 & $-9 \cdot 349$ & 13.491 & 0.352 \\
\hline 47 & 40.339 & 0.393 & 2.191 & -4.531 & 2.749 & -1.577 & $3 \cdot 340$ & 12.783 & -9.251 & $13 \cdot 561$ & 0.361 \\
\hline 48 & 40.248 & 0.302 & 2.322 & -4.474 & 2.607 & -1.590 & $3 \cdot 335$ & 12.983 & -9.239 & 13.635 & 0.373 \\
\hline
\end{tabular}




\begin{tabular}{|c|c|c|c|c|c|c|c|c|c|c|c|}
\hline 49 & 40.118 & 0.259 & 2.385 & -4.461 & 2.532 & -1.578 & $3 \cdot 370$ & 13.119 & -9.324 & 13.705 & 0.386 \\
\hline 50 & 40.030 & 0.272 & 2.344 & -4.453 & 2.549 & -1.541 & 3.435 & 13.167 & -9.465 & 13.763 & 0.402 \\
\hline 51 & 39.962 & 0.330 & 2.183 & -4.484 & 2.657 & -1.479 & $3 \cdot 531$ & 13.098 & -9.673 & 13.813 & 0.418 \\
\hline 52 & 39.918 & 0.410 & 1.960 & $-4 \cdot 511$ & 2.805 & -1.406 & 3.641 & 12.984 & -9.918 & 13.853 & 0.435 \\
\hline 53 & 39.838 & 0.493 & 1.750 & -4.542 & 2.969 & -1.335 & 3.742 & 12.864 & -10.181 & 13.889 & 0.451 \\
\hline 54 & $39 \cdot 742$ & 0.548 & 1.607 & -4.592 & 3.098 & -1.279 & 3.815 & 12.790 & -10.416 & 13.927 & 0.466 \\
\hline 55 & 39.608 & 0.579 & 1.582 & -4.648 & 3.183 & -1.241 & 3.863 & 12.830 & -10.609 & 13.969 & 0.479 \\
\hline 56 & 39.461 & 0.579 & 1.637 & -4.699 & 3.206 & -1.227 & 3.876 & 12.903 & -10.746 & 14.008 & 0.488 \\
\hline 57 & $39 \cdot 335$ & 0.561 & 1.754 & -4.780 & 3.205 & -1.231 & 3.853 & 12.996 & -10.826 & 14.027 & 0.492 \\
\hline $5^{8}$ & $39 \cdot 313$ & 0.551 & 1.910 & -4.871 & 3.201 & -1.242 & 3.813 & 13.101 & -10.821 & 14.038 & 0.494 \\
\hline 59 & 39.405 & 0.534 & 2.023 & -4.901 & 3.193 & -1.255 & $3 \cdot 773$ & 13.146 & -10.788 & 14.025 & 0.492 \\
\hline $6 o$ & $39 \cdot 596$ & 0.549 & 2.114 & -4.953 & 3.215 & -1.269 & $3 \cdot 719$ & 13.115 & -10.717 & 13.985 & 0.485 \\
\hline 61 & 39.834 & 0.514 & 2.212 & -4.994 & 3.225 & -1.271 & 3.648 & 13.079 & -10.637 & 13.946 & 0.481 \\
\hline 62 & 40.035 & 0.527 & 2.341 & -4.999 & 3.218 & -1.290 & $3 \cdot 594$ & 13.014 & -10.574 & 13.891 & 0.471 \\
\hline 63 & 40.148 & 0.522 & 2.515 & -5.014 & 3.196 & -1.313 & 3.535 & 12.992 & -10.547 & 13.837 & 0.461 \\
\hline 64 & 40.100 & 0.518 & 2.705 & -5.026 & 3.141 & -1.342 & 3.468 & 12.976 & -10.581 & 13.788 & 0.449 \\
\hline 65 & 39.920 & 0.521 & 2.877 & -5.048 & 3.103 & -1.364 & 3.413 & 12.953 & -10.693 & 13.732 & 0.438 \\
\hline 66 & 39.654 & 0.561 & 2.998 & -5.078 & 3.086 & -1.364 & 3.390 & 12.881 & -10.867 & 13.679 & 0.430 \\
\hline 67 & $39 \cdot 343$ & 0.633 & 2.994 & -5.141 & 3.127 & -1.349 & $3 \cdot 399$ & 12.720 & -11.108 & 13.610 & 0.421 \\
\hline 68 & 39.054 & 0.763 & 2.837 & -5.220 & 3.226 & -1.299 & 3.448 & 12.424 & -11.392 & 13.532 & 0.414 \\
\hline 69 & 38.807 & 0.921 & 2.566 & $-5 \cdot 312$ & $3 \cdot 378$ & -1.228 & 3.538 & 12.065 & -11.697 & 13.453 & 0.410 \\
\hline 70 & 38.608 & 1.079 & 2.271 & $-5 \cdot 399$ & $3 \cdot 502$ & -1.150 & 3.645 & 11.691 & -11.971 & 13.374 & 0.406 \\
\hline 71 & 38.441 & 1.184 & 2.049 & -5.419 & $3 \cdot 567$ & -1.091 & 3.733 & 11.415 & -12.158 & $13 \cdot 318$ & 0.403 \\
\hline 72 & 38.369 & 1.191 & 1.994 & -5.382 & $3 \cdot 553$ & -1.063 & 3.790 & 11.358 & -12.148 & 13.306 & 0.406 \\
\hline 73 & 38.412 & 1.061 & 2.220 & -5.247 & $3 \cdot 356$ & -1.078 & 3.796 & 11.639 & -11.913 & $13 \cdot 35^{2}$ & 0.414 \\
\hline 74 & 38.658 & 0.778 & 2.718 & -4.974 & 2.977 & -1.155 & $3 \cdot 718$ & 12.191 & -11.380 & 13.441 & 0.422 \\
\hline 75 & 39.101 & 0.384 & 3.455 & -4.611 & 2.455 & -1.276 & 3.592 & 12.968 & -10.610 & $13 \cdot 571$ & 0.433 \\
\hline 76 & 39.699 & -0.036 & 4.203 & -4.190 & 1.903 & -1.410 & 3.437 & 13.720 & -9.715 & 13.691 & 0.440 \\
\hline 77 & 40.354 & -0.396 & 4.863 & -3.812 & 1.414 & -1.521 & 3.305 & $14 \cdot 342$ & -8.861 & 13.793 & 0.446 \\
\hline 78 & 40.952 & -0.642 & $5 \cdot 303$ & -3.562 & 1.071 & -1.591 & 3.229 & $14 \cdot 741$ & -8.150 & 13.867 & $0.45^{2}$ \\
\hline 79 & 41.398 & -0.800 & $5 \cdot 565$ & -3.413 & 0.838 & -1.613 & 3.205 & 14.946 & -7.598 & 13.909 & $0.45^{8}$ \\
\hline 80 & 41.640 & -0.893 & $5 \cdot 762$ & -3.364 & o.677 & -1.606 & 3.225 & 15.073 & -7.243 & 13.948 & 0.466 \\
\hline 81 & 41.680 & -0.952 & 5.892 & -3.382 & 0.574 & -1.592 & 3.255 & 15.152 & -7.016 & 13.972 & 0.473 \\
\hline 82 & 41.516 & -0.995 & 6.005 & -3.425 & 0.496 & -1.571 & 3.285 & 15.180 & -6.924 & 13.986 & 0.477 \\
\hline 83 & 41.147 & -0.978 & 6.021 & -3.534 & 0.504 & -1.519 & $3 \cdot 357$ & 15.141 & -7.011 & 13.999 & 0.485 \\
\hline 84 & 40.544 & -0.836 & 5.774 & -3.797 & 0.706 & -1.417 & 3.485 & 14.829 & $-7 \cdot 377$ & 13.965 & 0.490 \\
\hline 85 & $39 \cdot 55^{8}$ & -0.420 & 4.984 & $-4 \cdot 389$ & 1.311 & -1.186 & $3 \cdot 771$ & 14.002 & -8.219 & 13.875 & 0.503 \\
\hline 86 & 38.032 & 0.378 & 3.399 & -5.480 & 2.493 & -0.768 & 4.287 & 12.465 & -9.769 & 13.689 & 0.521 \\
\hline 87 & 36.053 & 1.494 & 1.170 & -6.966 & 4.157 & -0.198 & 5.002 & 10.314 & -11.892 & 13.433 & 0.548 \\
\hline 88 & 34.278 & 2.520 & -0.921 & -8.260 & 5.686 & 0.315 & 5.642 & 8.263 & -13.816 & 13.174 & 0.565 \\
\hline 89 & 33.106 & 3.171 & -2.264 & -9.020 & 6.648 & 0.615 & 6.020 & 6.897 & -15.007 & 12.981 & 0.566 \\
\hline 90 & 32.353 & $3 \cdot 518$ & -3.010 & -9.422 & 7.189 & 0.773 & 6.217 & 6.178 & -15.685 & 12.876 & 0.564 \\
\hline 91 & 31.740 & $3 \cdot 731$ & -3.428 & $-9 \cdot 787$ & $7 \cdot 553$ & 0.867 & 6.345 & 5.873 & -16.159 & 12.836 & 0.566 \\
\hline 92 & 31.201 & 3.867 & -3.691 & -10.160 & 7.835 & 0.938 & 6.455 & 5.849 & -16.535 & 12.853 & 0.578 \\
\hline 93 & 30.965 & 3.889 & -3.715 & -10.428 & $7 \cdot 975$ & 0.956 & 6.491 & 6.107 & -16.617 & 12.920 & 0.593 \\
\hline 94 & 32.757 & 2.899 & -1.771 & -9.173 & 6.579 & 0.446 & 5.827 & 8.081 & -14.473 & 13.168 & 0.569 \\
\hline 95 & 35.264 & 1.614 & 0.659 & -7.420 & 4.807 & -0.210 & 4.959 & 10.462 & -11.614 & 13.451 & 0.534 \\
\hline 96 & 32.798 & 3.087 & -2.225 & -9.903 & 7.186 & 0.611 & 6.030 & 8.295 & -14.423 & 13.307 & 0.623 \\
\hline 97 & 32.350 & 3.464 & -2.956 & -10.672 & 7.869 & 0.844 & 6.333 & 7.891 & -14.935 & 13.325 & 0.661 \\
\hline 98 & 32.115 & 3.665 & -3.290 & -11.138 & 8.244 & 0.978 & 6.506 & $7 \cdot 768$ & -15.161 & 13.374 & 0.691 \\
\hline 99 & 31.836 & 3.783 & -3.449 & -11.486 & 8.476 & 1.072 & 6.638 & $7 \cdot 722$ & $-15 \cdot 345$ & 13.419 & 0.714 \\
\hline 100 & 31.406 & 3.880 & -3.557 & -11.816 & 8.620 & 1.146 & 6.760 & 7.684 & -15.653 & 13.457 & 0.733 \\
\hline 101 & 31.021 & 3.950 & $-3 \cdot 576$ & -12.023 & 8.675 & 1.201 & 6.857 & 7.654 & -15.927 & 13.482 & 0.746 \\
\hline 102 & 30.770 & 4.007 & -3.644 & -12.107 & 8.669 & 1.239 & 6.923 & $7 \cdot 595$ & -16.068 & 13.490 & 0.751 \\
\hline 103 & 30.791 & 4.013 & -3.635 & -12.026 & 8.531 & 1.230 & 6.926 & $7 \cdot 521$ & -15.972 & 13.476 & 0.745 \\
\hline 104 & 31.049 & 4.003 & -3.616 & -11.776 & 8.303 & 1.189 & 6.875 & $7 \cdot 454$ & -15.666 & 13.438 & 0.729 \\
\hline 105 & 31.515 & 3.899 & -3.484 & -11.311 & 7.905 & 1.082 & 6.734 & 7.469 & -15.147 & 13.389 & 0.698 \\
\hline
\end{tabular}




\begin{tabular}{|c|c|c|c|c|c|c|c|c|c|c|c|}
\hline 106 & 32.247 & 3.622 & -2.967 & -10.573 & 7.238 & 0.877 & 6.460 & 7.813 & -14.314 & $13 \cdot 355$ & 0.659 \\
\hline 107 & $33 \cdot 361$ & 3.041 & -1.827 & -9.445 & 6.143 & 0.512 & $5 \cdot 985$ & 8.713 & -13.035 & 13.388 & 0.610 \\
\hline 108 & 34.659 & 2.244 & -0.253 & -8.074 & $4 \cdot 787$ & 0.056 & $5 \cdot 397$ & 10.036 & -11.502 & 13.482 & 0.561 \\
\hline 109 & 35.005 & 1.896 & 0.477 & -7.449 & 4.117 & -0.155 & 5.131 & 10.620 & -11.033 & 13.498 & 0.532 \\
\hline 110 & 36.021 & 1.174 & 1.954 & -6.340 & 2.988 & -0.541 & 4.633 & 11.868 & -9.849 & 13.618 & 0.499 \\
\hline 111 & 37.651 & 0.151 & 3.939 & -4.846 & 1.525 & -1.050 & 3.969 & 13.579 & -8.075 & 13.820 & 0.466 \\
\hline 112 & 39.922 & -1.143 & 6.365 & -2.994 & -0.207 & -1.660 & 3.158 & 15.708 & -5.680 & 14.092 & 0.435 \\
\hline 113 & 42.002 & -2.208 & 8.324 & -1.432 & -1.592 & -2.150 & 2.498 & 17.437 & -3.619 & 14.336 & 0.415 \\
\hline 114 & $43 \cdot 589$ & -2.931 & 9.682 & -0.410 & -2.525 & -2.467 & 2.054 & 18.618 & -2.168 & 14.517 & 0.406 \\
\hline 115 & 44.293 & -3.185 & 10.153 & -0.063 & -2.817 & -2.578 & 1.899 & 19.026 & -1.681 & 14.611 & 0.407 \\
\hline 116 & 44.556 & -3.248 & 10.331 & -0.031 & -2.850 & -2.594 & 1.872 & 19.175 & -1.637 & 14.679 & 0.417 \\
\hline 117 & 44.456 & -3.232 & 10.346 & -0.171 & -2.796 & -2.562 & 1.931 & 19.222 & -1.904 & 14.751 & 0.431 \\
\hline 118 & 44.086 & -3.144 & 10.244 & -0.437 & -2.615 & -2.494 & 2.045 & 19.154 & -2.371 & 14.807 & 0.449 \\
\hline 119 & 43.652 & -3.058 & 10.124 & -0.705 & -2.428 & -2.440 & 2.156 & 19.049 & -2.865 & 14.854 & 0.461 \\
\hline 120 & $43 \cdot 318$ & -3.005 & 9.951 & -0.891 & -2.295 & -2.389 & 2.268 & 18.936 & -3.242 & 14.893 & 0.470 \\
\hline 121 & 43.250 & -2.983 & 9.833 & -0.942 & -2.232 & -2.363 & 2.350 & 18.845 & -3.407 & 14.929 & 0.475 \\
\hline 122 & $43 \cdot 510$ & -3.013 & 9.706 & -0.818 & -2.263 & -2.369 & 2.395 & 18.754 & $-3 \cdot 311$ & 14.958 & 0.472 \\
\hline 123 & 43.969 & -3.097 & 9.628 & -0.516 & -2.357 & -2.408 & 2.404 & 18.651 & -3.037 & 14.976 & 0.463 \\
\hline 124 & 44.977 & -3.460 & 10.064 & 0.620 & -2.565 & -2.614 & 2.484 & 18.578 & -2.220 & 15.054 & 0.437 \\
\hline 125 & 45.146 & -3.493 & 9.818 & 0.867 & -2.541 & -2.622 & 2.584 & 18.285 & -2.096 & 15.052 & 0.427 \\
\hline 126 & 44.985 & $-3 \cdot 515$ & $9 \cdot 581$ & 0.947 & -2.592 & -2.609 & 2.676 & 18.102 & -2.119 & 15.057 & 0.421 \\
\hline 127 & $44 \cdot 561$ & -3.529 & $9 \cdot 364$ & 0.941 & -2.642 & -2.597 & 2.762 & 17.976 & -2.253 & 15.061 & 0.416 \\
\hline 128 & 43.930 & -3.539 & 9.143 & 0.867 & -2.703 & -2.579 & 2.860 & 17.878 & -2.512 & 15.073 & 0.412 \\
\hline 129 & $43 \cdot 349$ & -3.544 & 9.011 & 0.749 & -2.769 & -2.562 & 2.958 & 17.836 & -2.734 & 15.087 & 0.411 \\
\hline 130 & 42.982 & -3.562 & 8.852 & 0.664 & -2.875 & -2.553 & 3.027 & 17.788 & -2.831 & 15.111 & 0.409 \\
\hline 131 & 42.947 & -3.599 & 8.702 & 0.670 & -3.023 & -2.557 & 3.063 & 17.745 & -2.719 & 15.141 & 0.408 \\
\hline 132 & 43.231 & -3.651 & 8.542 & 0.764 & -3.203 & -2.569 & 3.062 & $17 \cdot 700$ & -2.393 & 15.171 & 0.406 \\
\hline 133 & 43.671 & -3.707 & 8.459 & 0.860 & -3.411 & -2.587 & 3.040 & $17 \cdot 721$ & $-1.95^{2}$ & 15.215 & 0.407 \\
\hline 134 & 44.033 & -3.763 & 8.426 & 0.887 & -3.639 & -2.602 & 3.002 & 17.840 & -1.543 & 15.270 & 0.411 \\
\hline 135 & 44.226 & -3.815 & 8.451 & 0.825 & -3.892 & -2.591 & 2.981 & 18.068 & -1.240 & $15 \cdot 355$ & 0.425 \\
\hline 136 & 44.248 & -3.817 & 8.415 & 0.751 & -3.973 & -2.596 & 2.995 & 18.030 & -1.092 & 15.401 & 0.429 \\
\hline 137 & 44.081 & -3.782 & 8.339 & 0.708 & -3.907 & -2.588 & 3.066 & 17.792 & -1.121 & 15.447 & 0.433 \\
\hline 138 & 43.809 & $-3.75^{0}$ & 8.289 & 0.579 & -3.883 & -2.563 & 3.140 & 17.626 & -1.264 & 15.490 & 0.441 \\
\hline 139 & $43 \cdot 557$ & -3.725 & 8.243 & 0.449 & -3.871 & -2.543 & 3.202 & 17.476 & -1.427 & $15 \cdot 542$ & 0.449 \\
\hline 140 & $43 \cdot 537$ & -3.702 & 8.175 & 0.377 & -3.865 & -2.513 & 3.263 & 17.338 & -1.488 & 15.600 & 0.461 \\
\hline 141 & 43.809 & -3.692 & 8.102 & 0.383 & -3.863 & -2.485 & $3 \cdot 301$ & 17.210 & -1.356 & 15.652 & 0.473 \\
\hline 142 & 44.290 & -3.692 & 8.038 & 0.392 & -3.855 & -2.455 & $3 \cdot 325$ & 17.100 & -1.134 & $15 \cdot 711$ & 0.487 \\
\hline 143 & 44.818 & -3.691 & $7 \cdot 994$ & 0.479 & -3.848 & -2.427 & $3 \cdot 322$ & 16.986 & -0.904 & 15.745 & 0.498 \\
\hline 144 & 45.206 & -3.706 & $7 \cdot 992$ & 0.578 & -3.852 & -2.399 & $3 \cdot 310$ & 16.887 & -0.818 & $15 \cdot 774$ & 0.510 \\
\hline 145 & $45 \cdot 357$ & $-3 \cdot 715$ & 8.063 & 0.597 & -3.834 & -2.366 & 3.290 & 16.836 & -0.895 & $15 \cdot 797$ & 0.523 \\
\hline 146 & 45.187 & -3.738 & 8.167 & 0.515 & -3.874 & -2.326 & 3.269 & 16.888 & -1.194 & 15.820 & 0.537 \\
\hline 147 & 44.366 & -3.584 & 8.036 & -0.362 & -3.993 & -2.135 & 3.229 & 17.174 & -2.007 & 15.852 & 0.577 \\
\hline 148 & 43.803 & -3.491 & 7.989 & -0.581 & -3.755 & -2.051 & 3.293 & 16.976 & -2.606 & 15.834 & 0.592 \\
\hline 149 & 43.294 & -3.397 & 7.928 & -0.745 & -3.496 & -1.979 & 3.346 & 16.760 & -3.188 & 15.805 & 0.602 \\
\hline 150 & 42.988 & $-3 \cdot 318$ & 7.860 & -0.867 & -3.259 & -1.913 & $3 \cdot 380$ & 16.520 & -3.611 & 15.769 & 0.609 \\
\hline 151 & 42.900 & -3.245 & $7 \cdot 794$ & -0.892 & -3.027 & -1.859 & $3 \cdot 394$ & 16.245 & -3.894 & $15 \cdot 726$ & 0.613 \\
\hline 152 & 42.965 & -3.180 & $7 \cdot 747$ & -0.892 & -2.799 & -1.811 & 3.404 & 16.050 & -4.045 & 15.688 & 0.618 \\
\hline 153 & 43.027 & -3.112 & 7.696 & -0.912 & -2.570 & -1.778 & 3.399 & 15.868 & -4.148 & 15.632 & 0.616 \\
\hline 154 & 43.018 & -3.035 & 7.654 & -1.019 & -2.337 & -1.744 & $3 \cdot 411$ & 15.742 & -4.223 & 15.584 & 0.616 \\
\hline 155 & 42.844 & -2.950 & 7.610 & -1.195 & -2.080 & -1.697 & $3.45^{8}$ & 15.697 & $-4 \cdot 351$ & 15.535 & 0.617 \\
\hline 156 & 42.501 & -2.847 & $7 \cdot 557$ & -1.470 & -1.837 & -1.643 & 3.535 & 15.665 & -4.554 & 15.493 & 0.617 \\
\hline 157 & 42.118 & -2.737 & $7 \cdot 494$ & -1.720 & -1.584 & -1.585 & 3.620 & 15.606 & $-4 \cdot 760$ & 15.455 & 0.619 \\
\hline 158 & 41.789 & -2.643 & $7 \cdot 417$ & -1.959 & -1.396 & -1.532 & 3.707 & $15 \cdot 516$ & -4.919 & 15.418 & 0.617 \\
\hline 159 & 41.674 & -2.581 & $7 \cdot 417$ & -2.086 & -1.274 & -1.500 & $3 \cdot 766$ & 15.421 & -4.949 & $15 \cdot 367$ & 0.609 \\
\hline 160 & 41.866 & -2.547 & $7 \cdot 496$ & -2.103 & -1.228 & -1.478 & 3.803 & 15.384 & -4.755 & $15 \cdot 341$ & 0.604 \\
\hline 161 & 42.242 & -2.565 & 7.644 & -2.037 & -1.263 & -1.467 & 3.825 & $15 \cdot 312$ & -4.447 & $15 \cdot 313$ & 0.598 \\
\hline 162 & 42.695 & -2.611 & 7.939 & -1.922 & -1.359 & -1.468 & 3.822 & $15 \cdot 391$ & -4.089 & $15 \cdot 308$ & 0.596 \\
\hline
\end{tabular}




$\begin{array}{llllllllllll}163 & 43.034 & -2.665 & 8.284 & -1.801 & -1.487 & -1.478 & 3.804 & 15.492 & -3.782 & 15.303 & 0.591 \\ 164 & 43.215 & -2.723 & 8.698 & -1.696 & -1.619 & -1.476 & 3.812 & 15.657 & -3.586 & 15.307 & 0.592 \\ 165 & 43.217 & -2.784 & 9.154 & -1.607 & -1.750 & -1.474 & 3.823 & 15.838 & -3.519 & 15.323 & 0.595 \\ 166 & 43.070 & -2.859 & 9.616 & -1.507 & -1.907 & -1.478 & 3.828 & 16.015 & -3.549 & 15.337 & 0.597 \\ 167 & 42.952 & -2.960 & 10.082 & -1.345 & -2.093 & -1.492 & 3.830 & 16.204 & -3.566 & 15.366 & 0.600 \\ 168 & 42.992 & -3.096 & 10.600 & -1.078 & -2.348 & -1.530 & 3.805 & 16.370 & -3.441 & 15.393 & 0.600 \\ 169 & 43.209 & -3.270 & 11.057 & -0.720 & -2.647 & -1.580 & 3.748 & 16.485 & -3.161 & 15.426 & 0.597 \\ 170 & 43.568 & -3.453 & 11.461 & -0.275 & -2.973 & -1.647 & 3.663 & 16.518 & -2.752 & 15.460 & 0.592 \\ 171 & 43.932 & -3.634 & 11.775 & 0.154 & -3.291 & -1.707 & 3.574 & 16.544 & -2.322 & 15.491 & 0.587 \\ 172 & 44.179 & -3.770 & 12.044 & 0.499 & -3.568 & -1.770 & 3.481 & 16.604 & -1.966 & 15.527 & 0.581 \\ 173 & 44.275 & -3.863 & 12.261 & 0.730 & -3.802 & -1.818 & 3.413 & 16.698 & -1.701 & 15.566 & 0.579 \\ 174 & 44.170 & -3.936 & 12.419 & 0.834 & -3.993 & -1.862 & 3.355 & 16.841 & -1.571 & 15.606 & 0.576 \\ 175 & 46.105 & -3.934 & 8.906 & 0.961 & -2.474 & -2.811 & 1.727 & 19.089 & -1.819 & 14.859 & 0.425 \\ 176 & 46.029 & -3.942 & 9.029 & 1.116 & -2.578 & -2.857 & 1.680 & 19.123 & -1.811 & 14.825 & 0.411\end{array}$

Table S2. ESP differences $V_{n}-V_{o}$ as plotted in Figure $3 B$ Main Text. Values rounded at 3 decimal digits.

\begin{tabular}{|c|c|c|c|c|c|c|c|c|c|c|c|}
\hline Time & Glu113 & Glu122 $^{\mathrm{H}}$ & Ser186 & Thr94 & Thru18 & Tyri91 & Tyr268 & Watı & Wat2 & Glu181 ${ }^{\mathrm{H}}$ & Tyr192 \\
\hline o & 0.000 & 0.000 & 0.000 & 0.000 & 0.000 & 0.000 & 0.000 & 0.000 & 0.000 & 0.000 & 0.000 \\
\hline 1 & -7.991 & 4.681 & -9.622 & $-7 \cdot 510$ & $7 \cdot 302$ & 2.508 & 3.437 & -7.134 & -9.406 & -0.606 & 0.243 \\
\hline 2 & -8.084 & $4 \cdot 701$ & -9.626 & $-7 \cdot 564$ & $7 \cdot 312$ & 2.516 & $3 \cdot 45^{1}$ & -7.121 & -9.483 & -0.606 & 0.244 \\
\hline 3 & -8.297 & $4 \cdot 771$ & -9.654 & $-7 \cdot 722$ & $7 \cdot 334$ & 2.529 & 3.472 & -7.137 & -9.636 & -0.633 & 0.241 \\
\hline 4 & -8.611 & 4.885 & -9.682 & -7.989 & $7 \cdot 373$ & 2.548 & 3.495 & -7.201 & -9.886 & -0.693 & 0.232 \\
\hline 5 & -8.904 & 5.064 & -9.787 & -8.310 & $7 \cdot 446$ & 2.581 & 3.520 & $-7 \cdot 344$ & -10.119 & -0.776 & 0.220 \\
\hline 6 & -8.983 & 5.215 & -9.790 & -8.525 & $7 \cdot 423$ & 2.580 & 3.474 & -7.469 & -10.196 & -0.879 & 0.200 \\
\hline 7 & -8.559 & 5.168 & -9.481 & -8.374 & 7.134 & 2.480 & 3.284 & $-7 \cdot 388$ & -9.795 & -0.980 & 0.167 \\
\hline 8 & $-7 \cdot 591$ & 4.834 & -8.705 & -7.767 & 6.526 & 2.265 & 2.953 & -6.912 & -8.816 & -1.004 & 0.135 \\
\hline 9 & $-6.45^{8}$ & 4.368 & $-7 \cdot 746$ & -6.924 & $5 \cdot 785$ & 1.996 & 2.579 & -6.273 & -7.658 & -0.997 & 0.103 \\
\hline 10 & -5.638 & 4.010 & -6.957 & -6.288 & 5.179 & 1.788 & 2.299 & -5.819 & -6.805 & -1.019 & 0.072 \\
\hline 11 & -5.192 & 3.788 & -6.374 & -5.858 & 4.731 & 1.638 & 2.108 & $-5 \cdot 524$ & -6.268 & -1.046 & 0.047 \\
\hline 12 & -4.950 & 3.618 & -5.867 & $-5 \cdot 542$ & $4 \cdot 373$ & 1.530 & 1.968 & -5.289 & -5.938 & -1.073 & 0.028 \\
\hline 13 & -4.897 & $3 \cdot 519$ & $-5 \cdot 517$ & $-5 \cdot 349$ & 4.136 & 1.464 & 1.896 & -5.188 & -5.817 & -1.118 & 0.011 \\
\hline 14 & -4.981 & $3 \cdot 546$ & $-5 \cdot 380$ & -5.299 & 4.105 & 1.467 & 1.909 & -5.262 & -5.886 & -1.156 & 0.006 \\
\hline 15 & -5.227 & 3.666 & $-5 \cdot 524$ & -5.398 & 4.258 & 1.536 & 2.003 & $-5 \cdot 585$ & -6.183 & -1.215 & 0.005 \\
\hline 16 & $-5 \cdot 522$ & 3.873 & -5.838 & $-5 \cdot 576$ & 4.556 & 1.645 & 2.143 & -6.107 & -6.588 & -1.307 & 0.004 \\
\hline 17 & -5.796 & 4.056 & -6.197 & -5.743 & 4.870 & 1.759 & 2.287 & -6.645 & -6.992 & -1.383 & 0.008 \\
\hline 18 & -5.993 & 4.157 & -6.445 & -5.783 & 5.093 & 1.836 & 2.374 & -7.108 & -7.261 & -1.450 & 0.008 \\
\hline 19 & -6.029 & 4.152 & -6.513 & -5.677 & 5.177 & 1.867 & 2.412 & $-7 \cdot 326$ & $-7 \cdot 322$ & -1.478 & 0.009 \\
\hline 20 & -6.137 & 4.108 & -6.458 & -5.608 & 5.209 & 1.871 & 2.411 & -7.423 & $-7 \cdot 377$ & -1.493 & 0.009 \\
\hline 21 & -6.325 & 4.106 & -6.468 & -5.650 & 5.245 & 1.882 & 2.418 & -7.486 & $-7 \cdot 516$ & -1.516 & 0.010 \\
\hline 22 & -6.653 & 4.205 & -6.608 & -5.881 & $5 \cdot 386$ & 1.928 & 2.474 & $-7 \cdot 579$ & -7.821 & -1.540 & 0.013 \\
\hline 23 & -7.018 & $4 \cdot 375$ & -6.823 & -6.262 & $5 \cdot 579$ & 1.989 & 2.543 & -7.755 & -8.215 & -1.588 & 0.015 \\
\hline 24 & -7.172 & 4.525 & -7.027 & -6.591 & 5.729 & 2.033 & 2.591 & -7.853 & -8.509 & -1.629 & 0.015 \\
\hline 25 & -7.136 & 4.637 & -7.113 & -6.835 & 5.810 & 2.043 & 2.594 & -7.860 & -8.639 & -1.665 & 0.012 \\
\hline 26 & -6.982 & 4.676 & -7.072 & -6.955 & 5.799 & 2.025 & 2.559 & $-7 \cdot 770$ & -8.681 & -1.676 & 0.010 \\
\hline 27 & -6.778 & 4.673 & -6.943 & -6.972 & $5 \cdot 737$ & 1.983 & 2.485 & -7.612 & -8.663 & -1.671 & 0.007 \\
\hline 28 & -6.623 & 4.628 & -6.729 & -6.909 & 5.602 & 1.915 & 2.383 & -7.440 & -8.636 & -1.680 & -0.003 \\
\hline 29 & -6.509 & 4.574 & -6.440 & -6.776 & $5 \cdot 470$ & 1.843 & 2.269 & -7.209 & -8.604 & -1.663 & -0.008 \\
\hline 30 & -6.464 & 4.489 & $-6.15^{8}$ & -6.604 & $5 \cdot 304$ & 1.767 & 2.152 & -7.000 & -8.597 & -1.646 & -0.015 \\
\hline 31 & -6.406 & 4.412 & -5.885 & -6.378 & 5.174 & 1.699 & 2.050 & -6.802 & -8.586 & -1.622 & -0.020 \\
\hline 32 & -6.330 & $4 \cdot 35^{2}$ & $-5 \cdot 712$ & -6.157 & 5.070 & 1.648 & 1.963 & -6.672 & -8.572 & -1.594 & -0.023 \\
\hline 33 & -6.199 & 4.297 & -5.621 & -5.936 & 5.001 & 1.605 & 1.897 & -6.587 & -8.539 & -1.577 & -0.027 \\
\hline 34 & -6.091 & 4.283 & -5.649 & $-5 \cdot 782$ & 4.975 & 1.588 & 1.857 & -6.607 & -8.542 & -1.558 & -0.029 \\
\hline 35 & -5.991 & $4 \cdot 304$ & -5.767 & -5.668 & 5.012 & 1.593 & 1.843 & -6.692 & -8.552 & -1.546 & -0.028 \\
\hline 36 & -6.011 & $4 \cdot 341$ & -5.973 & -5.633 & 5.052 & 1.600 & 1.842 & -6.855 & -8.601 & -1.553 & -0.033 \\
\hline 37 & -6.109 & 4.389 & -6.188 & -5.653 & 5.069 & 1.617 & 1.841 & -7.017 & -8.634 & -1.555 & -0.035 \\
\hline 38 & -6.275 & 4.440 & -6.400 & -5.747 & 5.110 & 1.635 & 1.849 & -7.169 & -8.667 & -1.555 & -0.037 \\
\hline
\end{tabular}




\begin{tabular}{|c|c|c|c|c|c|c|c|c|c|c|c|}
\hline 39 & $-6.45^{2}$ & 4.460 & -6.582 & -5.834 & 5.104 & 1.646 & 1.849 & -7.255 & -8.623 & -1.551 & -0.039 \\
\hline 40 & -6.545 & 4.462 & -6.733 & -5.901 & 5.047 & 1.638 & 1.830 & $-7 \cdot 300$ & -8.517 & $-1.55^{2}$ & -0.044 \\
\hline 41 & -6.521 & 4.437 & -6.862 & -5.932 & $4 \cdot 974$ & 1.631 & 1.811 & $-7 \cdot 349$ & -8.334 & -1.542 & -0.048 \\
\hline 42 & -6.357 & $4 \cdot 393$ & -6.956 & $-5 \cdot 930$ & 4.867 & 1.615 & 1.775 & $-7 \cdot 336$ & -8.067 & -1.532 & -0.052 \\
\hline 43 & -6.104 & $4 \cdot 352$ & -7.021 & -5.921 & 4.767 & 1.603 & 1.747 & -7.269 & -7.755 & -1.506 & -0.051 \\
\hline 44 & -5.892 & 4.277 & -7.009 & -5.883 & 4.611 & 1.578 & 1.712 & -7.162 & -7.469 & -1.470 & -0.050 \\
\hline 45 & -5.707 & 4.193 & -6.947 & -5.832 & 4.450 & 1.552 & 1.677 & -6.991 & -7.195 & -1.420 & -0.046 \\
\hline 46 & -5.633 & 4.073 & -6.821 & $-5 \cdot 765$ & 4.254 & 1.524 & 1.645 & -6.778 & -7.006 & -1.349 & -0.038 \\
\hline 47 & -5.658 & $3 \cdot 981$ & -6.670 & -5.696 & 4.093 & 1.504 & 1.626 & -6.551 & -6.908 & -1.279 & -0.029 \\
\hline 48 & -5.749 & 3.890 & -6.538 & -5.639 & 3.950 & 1.490 & 1.621 & -6.351 & -6.896 & -1.206 & -0.018 \\
\hline 49 & -5.879 & 3.846 & -6.475 & -5.626 & 3.876 & 1.502 & 1.656 & -6.215 & -6.981 & -1.135 & -0.004 \\
\hline 50 & -5.967 & 3.859 & -6.517 & -5.618 & 3.892 & 1.539 & 1.721 & -6.167 & -7.122 & -1.078 & 0.012 \\
\hline 51 & -6.035 & 3.918 & -6.678 & -5.650 & 4.000 & 1.601 & 1.817 & -6.236 & $-7 \cdot 330$ & -1.028 & 0.028 \\
\hline 52 & -6.079 & 3.998 & -6.900 & -5.677 & 4.148 & 1.674 & 1.927 & -6.350 & $-7 \cdot 575$ & -0.988 & 0.045 \\
\hline 53 & -6.159 & 4.081 & -7.111 & -5.708 & $4 \cdot 313$ & 1.746 & 2.028 & -6.470 & -7.838 & -0.951 & 0.061 \\
\hline 54 & -6.255 & 4.136 & -7.254 & $-5.75^{8}$ & 4.441 & 1.802 & 2.101 & -6.544 & -8.073 & -0.914 & 0.076 \\
\hline 55 & -6.389 & 4.167 & -7.279 & -5.813 & $4 \cdot 526$ & 1.840 & 2.149 & -6.504 & -8.266 & -0.872 & 0.089 \\
\hline 56 & -6.536 & 4.166 & -7.223 & -5.865 & $4 \cdot 549$ & 1.854 & 2.162 & -6.431 & -8.403 & -0.833 & 0.098 \\
\hline 57 & -6.662 & 4.148 & -7.107 & -5.946 & $4 \cdot 549$ & 1.850 & 2.139 & -6.338 & -8.483 & -0.814 & 0.102 \\
\hline 58 & -6.684 & 4.138 & -6.951 & -6.037 & $4 \cdot 545$ & 1.839 & 2.099 & -6.233 & -8.478 & -0.803 & 0.104 \\
\hline 59 & -6.592 & 4.122 & -6.838 & -6.067 & 4.536 & 1.825 & 2.059 & -6.188 & -8.445 & -0.816 & 0.102 \\
\hline 60 & -6.401 & 4.137 & -6.747 & -6.119 & $4 \cdot 558$ & 1.811 & 2.004 & -6.219 & -8.374 & -0.856 & 0.095 \\
\hline 61 & -6.164 & 4.102 & -6.648 & $-6.16 o$ & $4 \cdot 568$ & 1.810 & 1.934 & -6.255 & -8.294 & -0.895 & 0.091 \\
\hline 62 & -5.962 & 4.114 & -6.520 & -6.165 & $4 \cdot 561$ & 1.791 & 1.879 & -6.320 & -8.231 & -0.949 & 0.081 \\
\hline 63 & -5.849 & 4.109 & -6.346 & -6.179 & $4 \cdot 540$ & 1.767 & 1.821 & -6.342 & -8.204 & -1.004 & 0.071 \\
\hline 64 & -5.897 & 4.105 & -6.156 & -6.192 & 4.484 & 1.738 & 1.754 & -6.358 & -8.238 & -1.053 & 0.059 \\
\hline 65 & -6.077 & 4.108 & -5.983 & -6.213 & 4.447 & 1.717 & 1.698 & -6.381 & -8.350 & -1.109 & 0.048 \\
\hline 66 & -6.343 & 4.148 & -5.862 & -6.244 & 4.429 & 1.716 & 1.676 & -6.453 & -8.524 & -1.162 & 0.040 \\
\hline 67 & -6.654 & 4.220 & -5.867 & -6.307 & 4.470 & 1.732 & 1.684 & -6.614 & -8.765 & -1.231 & 0.031 \\
\hline 68 & -6.943 & $4 \cdot 350$ & -6.024 & -6.386 & 4.570 & 1.782 & 1.734 & -6.910 & -9.049 & -1.309 & 0.024 \\
\hline 69 & -7.190 & 4.508 & -6.295 & -6.478 & 4.722 & 1.853 & 1.824 & -7.269 & -9.354 & -1.388 & 0.020 \\
\hline 70 & $-7 \cdot 390$ & 4.666 & -6.590 & -6.565 & 4.845 & 1.931 & 1.931 & -7.643 & -9.628 & -1.467 & 0.016 \\
\hline 71 & $-7 \cdot 557$ & $4 \cdot 771$ & -6.812 & -6.584 & 4.911 & 1.989 & 2.019 & -7.919 & -9.814 & -1.523 & 0.013 \\
\hline 72 & -7.628 & 4.778 & -6.867 & -6.548 & 4.896 & 2.018 & 2.076 & -7.976 & -9.805 & -1.534 & 0.016 \\
\hline 73 & -7.585 & 4.648 & -6.640 & -6.413 & 4.699 & 2.003 & 2.082 & -7.695 & -9.570 & -1.489 & 0.024 \\
\hline 74 & $-7 \cdot 339$ & $4 \cdot 365$ & -6.142 & -6.139 & $4 \cdot 321$ & 1.925 & 2.004 & -7.143 & -9.037 & -1.400 & 0.032 \\
\hline 75 & -6.896 & $3 \cdot 971$ & -5.406 & $-5 \cdot 776$ & $3 \cdot 799$ & 1.805 & 1.878 & -6.366 & -8.267 & -1.270 & 0.043 \\
\hline 76 & -6.298 & $3 \cdot 55^{2}$ & -4.657 & $-5 \cdot 355$ & 3.246 & 1.671 & 1.723 & -5.614 & $-7 \cdot 372$ & -1.150 & 0.050 \\
\hline 77 & -5.643 & 3.192 & -3.997 & -4.977 & 2.757 & 1.560 & 1.591 & -4.992 & -6.518 & -1.048 & 0.056 \\
\hline 78 & -5.045 & 2.946 & $-3 \cdot 55^{8}$ & -4.727 & 2.414 & 1.490 & 1.515 & -4.593 & -5.807 & -0.974 & 0.062 \\
\hline 79 & -4.599 & 2.787 & -3.295 & -4.578 & 2.182 & 1.468 & 1.491 & $-4 \cdot 388$ & -5.255 & -0.932 & 0.068 \\
\hline 80 & $-4 \cdot 357$ & 2.695 & -3.099 & $-4 \cdot 529$ & 2.021 & 1.474 & 1.511 & -4.261 & -4.900 & -0.893 & 0.076 \\
\hline 81 & $-4 \cdot 317$ & 2.636 & -2.969 & -4.547 & 1.917 & 1.489 & 1.540 & -4.182 & -4.673 & -0.869 & 0.083 \\
\hline 82 & -4.481 & 2.592 & -2.856 & -4.590 & 1.840 & 1.510 & 1.571 & -4.154 & $-4 \cdot 581$ & -0.855 & 0.087 \\
\hline 83 & -4.851 & 2.610 & -2.840 & -4.699 & 1.847 & 1.561 & 1.642 & -4.193 & -4.668 & -0.841 & 0.095 \\
\hline 84 & -5.453 & 2.752 & -3.087 & -4.963 & 2.049 & 1.663 & 1.771 & -4.505 & -5.034 & -0.876 & 0.100 \\
\hline 85 & -6.439 & 3.167 & -3.877 & -5.555 & 2.654 & 1.895 & 2.057 & -5.332 & -5.876 & -0.966 & 0.112 \\
\hline 86 & -7.965 & 3.965 & -5.462 & -6.646 & 3.837 & 2.313 & 2.573 & -6.869 & -7.426 & -1.152 & 0.131 \\
\hline 87 & -9.944 & 5.082 & -7.691 & -8.131 & $5 \cdot 501$ & 2.883 & 3.288 & -9.020 & -9.549 & -1.407 & 0.158 \\
\hline 88 & -11.719 & 6.107 & -9.782 & -9.425 & 7.029 & 3.395 & 3.928 & -11.071 & -11.473 & -1.667 & 0.175 \\
\hline 89 & -12.891 & 6.758 & -11.124 & -10.185 & $7 \cdot 991$ & 3.696 & 4.306 & -12.437 & -12.664 & -1.860 & 0.176 \\
\hline 90 & -13.644 & 7.105 & -11.870 & -10.588 & 8.532 & 3.854 & 4.503 & -13.156 & $-13 \cdot 342$ & -1.965 & 0.174 \\
\hline 91 & -14.257 & $7 \cdot 318$ & -12.289 & -10.952 & 8.896 & $3 \cdot 948$ & 4.631 & -13.461 & -13.816 & -2.004 & 0.176 \\
\hline 92 & -14.796 & $7 \cdot 454$ & $-12.55^{2}$ & -11.326 & 9.179 & 4.019 & $4 \cdot 741$ & -13.485 & -14.192 & -1.988 & 0.187 \\
\hline 93 & -15.032 & $7 \cdot 476$ & -12.575 & -11.593 & $9 \cdot 319$ & 4.037 & $4 \cdot 777$ & -13.227 & -14.274 & -1.921 & 0.203 \\
\hline 94 & -13.240 & 6.486 & -10.631 & -10.338 & $7 \cdot 922$ & $3 \cdot 5^{26}$ & 4.113 & -11.253 & -12.129 & -1.673 & 0.179 \\
\hline 95 & -10.733 & 5.202 & -8.202 & -8.585 & 6.150 & 2.871 & 3.245 & -8.872 & -9.271 & -1.389 & 0.144 \\
\hline
\end{tabular}




\begin{tabular}{|c|c|c|c|c|c|c|c|c|c|c|c|}
\hline 96 & -13.199 & 6.674 & -11.085 & -11.068 & 8.529 & 3.692 & $4 \cdot 316$ & -11.039 & -12.080 & -1.534 & 0.233 \\
\hline 97 & -13.647 & 7.052 & -11.817 & -11.837 & 9.212 & 3.924 & 4.619 & -11.443 & -12.592 & -1.516 & 0.271 \\
\hline 98 & -13.883 & 7.253 & $-12.15^{1}$ & -12.303 & $9 \cdot 587$ & 4.059 & 4.792 & -11.566 & -12.818 & -1.467 & 0.301 \\
\hline 99 & -14.161 & $7 \cdot 370$ & -12.309 & -12.651 & 9.819 & 4.152 & 4.924 & -11.612 & -13.002 & -1.422 & 0.324 \\
\hline 100 & -14.591 & $7 \cdot 467$ & -12.418 & -12.982 & $9 \cdot 963$ & 4.227 & 5.046 & -11.649 & $-13 \cdot 310$ & -1.384 & 0.342 \\
\hline 101 & -14.977 & $7 \cdot 537$ & -12.437 & -13.188 & 10.019 & 4.282 & 5.143 & -11.680 & -13.584 & -1.359 & 0.356 \\
\hline 102 & -15.228 & $7 \cdot 595$ & -12.505 & -13.272 & 10.012 & $4 \cdot 319$ & 5.209 & -11.739 & $-13 \cdot 725$ & -1.351 & 0.361 \\
\hline 103 & -15.206 & 7.601 & -12.495 & -13.191 & 9.874 & $4 \cdot 310$ & 5.212 & -11.813 & -13.629 & -1.365 & 0.355 \\
\hline 104 & -14.948 & $7 \cdot 591$ & -12.477 & -12.942 & 9.646 & 4.270 & 5.161 & -11.880 & $-13 \cdot 323$ & -1.403 & 0.339 \\
\hline 105 & -14.482 & $7 \cdot 486$ & -12.345 & -12.476 & 9.249 & 4.163 & 5.020 & -11.865 & -12.804 & $-1.45^{2}$ & 0.308 \\
\hline 106 & $-13 \cdot 750$ & 7.209 & -11.828 & -11.739 & 8.581 & 3.957 & 4.746 & -11.521 & -11.971 & -1.486 & 0.269 \\
\hline 107 & -12.636 & 6.628 & -10.687 & -10.610 & $7 \cdot 486$ & 3.593 & 4.271 & -10.621 & -10.692 & -1.453 & 0.220 \\
\hline 108 & -11.339 & 5.831 & -9.113 & -9.240 & 6.130 & 3.137 & 3.683 & -9.298 & -9.159 & -1.359 & 0.171 \\
\hline 109 & -10.992 & $5 \cdot 484$ & -8.384 & -8.614 & 5.460 & 2.925 & 3.416 & -8.714 & -8.690 & -1.342 & 0.142 \\
\hline 110 & -9.976 & $4 \cdot 761$ & -6.906 & -7.505 & $4 \cdot 331$ & 2.539 & 2.919 & $-7 \cdot 466$ & $-7 \cdot 506$ & -1.223 & 0.109 \\
\hline 111 & -8.346 & 3.738 & -4.922 & -6.012 & 2.868 & 2.030 & 2.255 & -5.755 & -5.732 & -1.021 & 0.076 \\
\hline 112 & -6.075 & 2.444 & -2.495 & -4.159 & 1.136 & 1.420 & 1.444 & -3.626 & -3.337 & -0.749 & 0.045 \\
\hline 113 & -3.995 & 1.379 & -0.537 & -2.597 & -0.249 & 0.931 & 0.784 & -1.897 & -1.276 & -0.505 & 0.025 \\
\hline 114 & -2.409 & 0.656 & 0.821 & -1.575 & -1.182 & 0.613 & 0.340 & -0.716 & 0.175 & -0.323 & 0.016 \\
\hline 115 & -1.704 & 0.403 & 1.292 & -1.229 & -1.474 & 0.502 & 0.185 & -0.308 & 0.662 & -0.230 & 0.017 \\
\hline 116 & -1.441 & 0.339 & 1.470 & -1.196 & -1.507 & 0.486 & 0.158 & -0.159 & 0.706 & -0.161 & 0.027 \\
\hline 117 & -1.541 & 0.356 & 1.486 & -1.336 & -1.453 & 0.518 & 0.217 & -0.112 & 0.439 & -0.090 & 0.041 \\
\hline 118 & -1.911 & 0.443 & 1.384 & -1.602 & -1.272 & 0.587 & 0.331 & -0.180 & -0.028 & -0.034 & 0.059 \\
\hline 119 & -2.345 & 0.529 & 1.263 & -1.871 & -1.085 & 0.640 & 0.441 & -0.285 & -0.522 & 0.013 & 0.071 \\
\hline 120 & -2.679 & 0.583 & 1.090 & -2.057 & -0.952 & 0.692 & 0.554 & -0.398 & -0.899 & 0.052 & 0.080 \\
\hline 121 & -2.747 & 0.605 & 0.973 & -2.108 & -0.888 & 0.718 & 0.636 & -0.489 & -1.064 & 0.089 & 0.085 \\
\hline 122 & -2.488 & 0.574 & 0.846 & -1.983 & -0.920 & 0.712 & 0.681 & -0.580 & -0.968 & 0.117 & 0.082 \\
\hline 123 & -2.029 & 0.491 & 0.767 & -1.681 & -1.014 & 0.672 & 0.690 & -0.683 & -0.694 & 0.135 & 0.073 \\
\hline 124 & -1.020 & 0.128 & 1.204 & -0.545 & -1.222 & 0.467 & 0.769 & -0.756 & 0.123 & 0.213 & 0.047 \\
\hline 125 & -0.851 & 0.095 & 0.958 & -0.298 & -1.197 & $0.45^{8}$ & 0.870 & -1.049 & 0.247 & 0.211 & 0.037 \\
\hline 126 & -1.013 & 0.072 & 0.721 & -0.218 & -1.249 & 0.471 & 0.961 & -1.232 & 0.224 & 0.216 & 0.031 \\
\hline 127 & -1.436 & 0.059 & 0.504 & -0.225 & -1.299 & 0.484 & 1.048 & -1.358 & 0.090 & 0.220 & 0.025 \\
\hline 128 & -2.068 & 0.049 & 0.283 & -0.298 & -1.360 & 0.501 & 1.146 & -1.456 & -0.169 & 0.233 & 0.022 \\
\hline 129 & -2.648 & 0.043 & 0.150 & -0.417 & -1.426 & 0.519 & 1.244 & -1.498 & -0.391 & 0.246 & 0.021 \\
\hline 130 & -3.015 & 0.025 & -0.009 & -0.501 & -1.531 & 0.527 & 1.313 & -1.546 & -0.488 & 0.271 & 0.019 \\
\hline 131 & -3.051 & -0.011 & -0.159 & -0.495 & -1.680 & 0.524 & 1.349 & -1.589 & -0.376 & 0.300 & 0.018 \\
\hline 132 & -2.766 & -0.064 & -0.318 & -0.401 & -1.860 & 0.511 & 1.348 & -1.634 & -0.050 & 0.330 & 0.016 \\
\hline 133 & -2.327 & -0.119 & -0.402 & -0.305 & -2.067 & 0.494 & 1.326 & -1.613 & 0.391 & 0.375 & 0.017 \\
\hline 134 & -1.964 & -0.176 & -0.434 & -0.279 & -2.296 & 0.479 & 1.288 & -1.494 & 0.800 & 0.429 & 0.021 \\
\hline 135 & -1.771 & -0.228 & -0.409 & -0.340 & -2.548 & 0.490 & 1.267 & -1.266 & 1.104 & 0.514 & 0.035 \\
\hline 136 & -1.749 & -0.230 & -0.445 & -0.415 & -2.629 & 0.485 & 1.281 & -1.304 & 1.251 & 0.560 & 0.039 \\
\hline 137 & -1.916 & -0.194 & -0.522 & $-0.45^{8}$ & -2.564 & 0.492 & 1.352 & -1.542 & 1.222 & 0.606 & 0.043 \\
\hline 138 & -2.188 & -0.163 & -0.571 & -0.587 & -2.540 & 0.517 & 1.426 & -1.708 & 1.079 & 0.650 & 0.051 \\
\hline 139 & -2.440 & -0.137 & -0.618 & -0.717 & -2.527 & 0.538 & 1.488 & -1.858 & 0.916 & 0.701 & 0.059 \\
\hline 140 & -2.460 & -0.115 & -0.686 & -0.789 & -2.522 & 0.567 & 1.549 & -1.996 & 0.855 & 0.760 & 0.071 \\
\hline 141 & -2.188 & -0.104 & -0.759 & -0.783 & -2.520 & 0.595 & 1.587 & -2.124 & 0.987 & 0.811 & 0.083 \\
\hline 142 & -1.708 & -0.105 & -0.823 & -0.774 & -2.512 & 0.626 & 1.611 & -2.234 & 1.209 & 0.870 & 0.097 \\
\hline 143 & -1.179 & -0.104 & -0.866 & -0.687 & -2.505 & 0.653 & 1.608 & -2.348 & 1.439 & 0.904 & 0.108 \\
\hline 144 & -0.791 & -0.119 & -0.868 & -0.588 & -2.508 & 0.682 & 1.596 & -2.447 & 1.525 & 0.933 & 0.120 \\
\hline 145 & -0.641 & -0.128 & -0.797 & -0.568 & -2.491 & 0.714 & 1.576 & -2.498 & 1.448 & 0.956 & 0.133 \\
\hline 146 & -0.810 & -0.151 & -0.693 & -0.650 & -2.530 & 0.754 & 1.555 & -2.446 & 1.149 & 0.979 & 0.147 \\
\hline 147 & -1.632 & 0.003 & -0.824 & -1.527 & -2.650 & 0.946 & 1.515 & -2.160 & 0.336 & 1.011 & 0.187 \\
\hline 148 & -2.194 & 0.096 & -0.871 & -1.747 & -2.412 & 1.030 & 1.579 & -2.358 & -0.263 & 0.993 & 0.202 \\
\hline 149 & -2.703 & 0.191 & -0.933 & -1.911 & -2.153 & 1.102 & 1.632 & -2.574 & -0.845 & 0.964 & 0.212 \\
\hline 150 & -3.009 & 0.269 & -1.000 & -2.032 & -1.916 & 1.168 & 1.665 & -2.814 & -1.268 & 0.928 & 0.219 \\
\hline 151 & -3.097 & 0.343 & -1.066 & -2.057 & -1.684 & 1.221 & 1.679 & -3.089 & -1.551 & 0.885 & 0.223 \\
\hline $15^{2}$ & -3.032 & 0.407 & -1.113 & -2.058 & -1.455 & 1.270 & 1.689 & -3.284 & -1.702 & 0.847 & 0.228 \\
\hline
\end{tabular}




\begin{tabular}{|c|c|c|c|c|c|c|c|c|c|c|c|}
\hline 153 & -2.970 & 0.475 & -1.165 & -2.078 & -1.227 & 1.303 & 1.685 & -3.466 & -1.805 & 0.791 & 0.226 \\
\hline 154 & -2.979 & 0.553 & -1.207 & -2.185 & -0.994 & 1.337 & 1.697 & -3.592 & -1.880 & 0.743 & 0.226 \\
\hline 155 & -3.153 & 0.637 & -1.250 & -2.360 & -0.737 & 1.383 & 1.743 & -3.637 & -2.008 & 0.694 & 0.227 \\
\hline 156 & -3.496 & 0.740 & -1.303 & -2.636 & -0.494 & 1.437 & 1.821 & -3.669 & -2.211 & 0.652 & 0.227 \\
\hline 157 & -3.879 & 0.851 & -1.367 & -2.886 & -0.240 & 1.496 & 1.906 & -3.728 & -2.417 & 0.614 & 0.229 \\
\hline 158 & -4.208 & 0.944 & -1.444 & -3.125 & -0.053 & 1.548 & 1.993 & -3.818 & -2.576 & 0.577 & 0.227 \\
\hline 159 & -4.323 & 1.007 & -1.444 & -3.251 & 0.069 & 1.580 & 2.052 & -3.913 & -2.606 & 0.526 & 0.219 \\
\hline 160 & -4.131 & 1.040 & -1.365 & -3.268 & 0.116 & 1.602 & 2.089 & -3.950 & -2.412 & 0.500 & 0.214 \\
\hline 161 & -3.755 & 1.022 & -1.217 & -3.202 & 0.080 & 1.613 & 2.111 & -4.022 & -2.104 & 0.472 & 0.208 \\
\hline 162 & -3.302 & 0.977 & -0.921 & -3.088 & -0.015 & 1.613 & 2.108 & -3.943 & -1.746 & 0.468 & 0.206 \\
\hline 163 & -2.964 & 0.922 & -0.576 & -2.967 & -0.143 & 1.602 & 2.090 & -3.842 & -1.439 & 0.463 & 0.201 \\
\hline 164 & -2.782 & 0.864 & -0.162 & -2.861 & -0.275 & 1.604 & 2.098 & -3.677 & -1.243 & 0.467 & 0.202 \\
\hline 165 & -2.780 & 0.804 & 0.294 & -2.772 & -0.407 & 1.606 & 2.108 & -3.496 & -1.176 & 0.482 & 0.205 \\
\hline 166 & -2.927 & 0.729 & 0.756 & -2.672 & -0.564 & 1.602 & 2.114 & -3.319 & -1.206 & 0.496 & 0.207 \\
\hline 167 & -3.045 & 0.627 & 1.221 & -2.510 & -0.750 & 1.589 & 2.116 & -3.130 & -1.223 & 0.525 & 0.210 \\
\hline 168 & -3.005 & 0.491 & 1.739 & -2.243 & -1.005 & 1.550 & 2.091 & -2.964 & -1.098 & 0.553 & 0.210 \\
\hline 169 & -2.788 & 0.318 & 2.197 & -1.886 & -1.304 & 1.500 & 2.034 & -2.849 & -0.818 & 0.585 & 0.207 \\
\hline 170 & -2.429 & 0.134 & 2.600 & -1.441 & -1.630 & 1.434 & 1.949 & -2.816 & -0.408 & 0.620 & 0.202 \\
\hline 171 & -2.065 & -0.046 & 2.915 & -1.012 & -1.948 & 1.374 & 1.860 & -2.790 & 0.021 & 0.650 & 0.197 \\
\hline 172 & -1.819 & -0.182 & 3.183 & -0.667 & -2.225 & 1.311 & 1.767 & -2.730 & 0.377 & 0.686 & 0.191 \\
\hline 173 & -1.722 & -0.276 & 3.400 & -0.436 & -2.459 & 1.263 & 1.699 & -2.636 & 0.642 & 0.725 & 0.189 \\
\hline 174 & -1.827 & -0.349 & 3.559 & -0.331 & -2.650 & 1.219 & 1.640 & -2.493 & 0.772 & 0.765 & 0.186 \\
\hline 175 & 0.108 & -0.347 & 0.046 & -0.204 & -1.131 & 0.269 & 0.013 & -0.245 & 0.524 & 0.018 & 0.035 \\
\hline 176 & 0.032 & -0.354 & 0.168 & -0.050 & -1.235 & 0.223 & -0.034 & -0.211 & 0.532 & -0.015 & 0.021 \\
\hline
\end{tabular}

\section{S4. $\mathbf{p K}_{\mathrm{a}}$ Calculations}

\section{S4.1 Theory}

All $\mathrm{pK}_{\mathrm{a}}$ calculations performed here correspond to predicting the titration behavior (or protonation probability) of the ionizable residues in the protein as a function of $\mathrm{pH}$. From the computed titration profile, the effective $\mathrm{pK}_{\mathrm{a}}$ value of a given residue corresponds to the half-protonation point, i.e., the $\mathrm{pH}$ at which the protonation probability of the residue is one-half. Assuming that the protonation probability is mostly of electrostatic nature ${ }^{23}$, the titration calculations rely on computing the electrostatic free energy for each of the many possible protonation states of the protein at a given $\mathrm{pH}$ and temperature (see below). Due to combinatorial explosion and the intrinsic difficulty of obtaining reliable free energies for large protein molecules, a self-consistent formalism for $\mathrm{pK}_{\mathrm{a}}$ calculations based on continuum electrostatics has been established ${ }^{3,24}$. In this framework, the protein atoms are described explicitly as a set of partial charges embedded in the low dielectric volume of the protein. The solvent is described implicitly as a high-dielectric medium. Mobile ions in solution (salts) are represented as a Boltzmann-distributed charge density. In the case of membrane proteins such as Rh, the effect of the lipids may also be modeled, for instance using a low-dieletric slab surrounding the protein; here, no membrane model was considered (see section $\mathrm{S}_{4} .3$ ). The electrostatic potentials of such an approximate system are computed numerically by solving the Poisson-Boltzmann equation (PBE) or its linearized form (LPBE) for low ionic strengths. This formalism, which is presented below and was used in this study, is exact within the framework of the $\mathrm{LPBE}^{25}$.

In a protein, the $\mathrm{pK}_{\mathrm{a}}$ of any ionizable residue is that of the titratable site(s) of its side chain (for convenience, the protein main-chain $\mathrm{N}$ - or $\mathrm{C}$ - terminal titratable groups are considered as individual residues). For most ionizable residues, a single site is titratable and two states are allowed: protonated or deprotonated. The Histidine residue has two titratable sites and three protonation states. For all titratable sites, a consistent set of charges is required to properly define the protonation states and compute the free energy differences between the protonated and deprotonated forms.

The $\mathrm{pK}_{\mathrm{a}}$ value of a residue in the protein can be significantly shifted compared to that of the isolated residue in solution (model compound) essentially for two reasons. First, embedding the titratable site in the relatively large low-dielectric volume of the protein causes destabilization of the charged form (usually found in solution at $\mathrm{pH}$ 7) because the solvent reaction field is decreased; buried residues in particular are likely to experience large shifts. Second, the titratable site in 
the protein is in interaction with the other charges and/or titratable sites, which can either stabilize of destabilize the charged form. As a matter of fact, the interaction of ionizable sites that titrate at similar $\mathrm{pH}$ ranges may lead to titration curves that deviate significantly from the standard sigmoidal behavior predicted by the Henderson-Hasselbalch equation $^{26}$.

For a titratable site $\mu$ of the protein, the perturbation due to the protein environment introduces a $\Delta p K_{a, \mu}$ shift such that

$$
p K_{a, \mu}=p K_{a, \mu}^{\text {model }}+\Delta p K_{a, \mu}
$$

where $p K_{a, \mu}$ is the effective pKa value of the residue embedded in the protein, $p K_{a, \mu}^{\text {model }}$ is the reference value for the model compound (the free residue in solution), which is typically about 4 for acids and 10 or above for bases; see Table 33 (column Std) for the reference values used in this study. Under the LPBE approximation, the $\Delta p K_{a}$ shift can be divided into three independent contributions:

$$
\Delta p K_{a, \mu}=-\frac{1}{R T \ln 10}\left(\Delta \Delta G_{\mu}^{\text {Born }}+\Delta \Delta G_{\mu}^{\text {back }}+\Delta \Delta G_{\mu}^{\text {inter }}\right)
$$

where the first two free energy differences describe the burial of the isolated titratable group within the protein, and the last term is the interaction energy with the other titratable groups; see ref. 23 for details on how to obtain these quantities from the electrostatic potentials. The $\Delta \Delta G$ corresponds to the difference in electrostatic free energy on moving the titratable site in its neutral versus charged form from solution to the protein interior. More specifically, $\Delta \Delta G_{\mu}^{B o r n}$ is the desolvation penalty for placing the titratable site (protonated or not) within the low-dielectric protein volume, which is often called the "self" energy. $\Delta \Delta G_{\mu}^{\text {back }}$ is the contribution arising from the interaction with the non-titrating or background charges of the protein, i.e., the stabilization/destabilization of the charged state due to the permanent electric field generated by the protein. $\Delta \Delta G_{\mu}^{\text {Born }}$ and $\Delta \Delta G_{\mu}^{b a c k}$ are both responsible for the strong shift that a titratable site may experience within the protein as compared to the isolated site in solution. Their contributions define the intrinsic $\mathrm{pK}_{\mathrm{a}}$ of the titratable site as

$$
p K_{a, \mu}^{i n t r}=p K_{a, \mu}^{m o d e l}-\frac{1}{R T \ln 10}\left(\Delta \Delta G_{\mu}^{\text {Born }}+\Delta \Delta G_{\mu}^{\text {back }}\right)
$$

which is the hypothetical $\mathrm{pK}_{\mathrm{a}}$ value that the titratable site possesses when all other titratable residues in the protein are in their neutral state (protonated for an acid, deprotonated for a base).

The $\Delta \Delta G_{\mu}^{\text {inter }}$ term accounts for the interaction between the titratable sites in every possible protonation micro-states of the protein averaged over the canonical ensemble at any given $\mathrm{pH}$. Because the calculation of this term involves a computational effort that rises exponentially with the number of titratable sites, in practice $\Delta \Delta G_{\mu}^{\text {inter }}$ is decomposed into additive pair-wise (site-site) contributions between the $N$ titratable sites of the protein. These energies are tabulated in a $W_{\mu \nu}$ matrix of Coulomb interactions between the sites $\mu$ and $v$ when they are in their charged form; see ref. 23 for their evaluation from the electrostatic potentials. The signed elements of $W_{\mu \nu}$ are non-zero when both $\mu$ and $v$ are not in their reference protonation state. For instance, a negative $W_{\mu \nu}$ element means that the charged states of the $\mu$, $v$ pair are stabilizing each other, that is, a deprotonated acid interacting with a protonated base. Conversely, a positive $W_{\mu \nu}$ element corresponds to two like-charged sites that destabilize each other. The interactions between the titratable sites may shift even more the effective $\mathrm{pK}_{\mathrm{a}}$ of a given site compared to its value in solution; at the same time, they may compensate for the shift due to $p K_{a, \mu}^{i n t r}$ such that, for instance, a buried acid becomes even more acidic in the presence of (buried) strong bases. Remarkably, the site-site interactions may perturb the titration profiles such that the interplay of ionizable residues titrating in similar $\mathrm{pH}$ ranges produce non-sigmoidal titration behavior ${ }^{26}$ or even undefined $\mathrm{pK}_{\mathrm{a}}$ values when multiple half-protonation points coexist.

At a given $\mathrm{pH}$ and temperature, the electrostatic free energy of a protonation micro-state $n$ of the protein containing $N$ titratable sites $(\mu \neq v)$ and $2^{N}$ protonation states is given as: 


$$
G^{n}=\sum_{\mu=1}^{N}\left(\left(x_{\mu}^{n}-z_{\mu}\right) R T \ln 10\left(p H-p K_{a, \mu}^{i n t r}\right)\right)+\frac{1}{2} \sum_{\mu=1}^{N} \sum_{v=1}^{N} W_{\mu \nu}
$$

where $x_{\mu}^{n} \in\{0,1\}$ represents the protonation state (deprotonated or protonated) of the titratable site in the current microstate $n$ of the protein; $z_{\mu} \in\{0,1\}$ expresses its acidic character (base or acid); and $p K_{a, \mu}^{\text {intr }}$ is its intrinsic $p K_{\mathrm{a}}$ as defined above. The $n$-th micro-state of the protein embedding $N$ titratable sites is defined by the protonation state vector $\vec{x}_{n}=\left(x_{1}^{n}, x_{2}^{n}, \ldots, x_{N}^{n}\right)$. The protonation probability $\left\langle x_{\mu}\right\rangle$ of the titratable site $\mu$ is then evaluated from the thermodynamic average over all possible micro-states of the protein as

$$
\left\langle x_{\mu}\right\rangle=\frac{\sum_{n=1}^{2^{N}} x_{\mu}^{n} e^{-G^{n} / R T}}{\sum_{n=1}^{2^{N}} e^{-G^{n} / R T}}
$$

For computational efficiency, the ensemble average above is approximated using several schemes ${ }^{23}$; here, we used a Metropolis Monte Carlo approach (see section $\mathrm{S}_{4.4}$ ). The effective $\mathrm{pK}_{\mathrm{a}}$ of the titratable site in the protein is finally estimated by computing the titration curve as a function of $\mathrm{pH}$ at the temperature of interest, and identify the $\mathrm{pH}$ value at which the protonation probability is one-half.

\section{S4.2 Structure preparation}

Four different structural models of visual Rh (atomic coordinates, charges and radii from the AMBER force-field ${ }^{2}$ ) taken along the computed retinal isomerization path and prepared in PQR format as described in section S3.1 were considered for the $\mathrm{pK}_{\mathrm{a}}$ calculations: (i) snapshot \#o, the ground-state structure at o fs; (ii) snapshot \#1, the vertical (Franck-Condon) excited-state of the retinal at $1 \mathrm{fs}$; (iii) snapshot \#92, a conformation near the conical intersection (CI) at 92 fs; and (iv) snapshot \#115, a Rh conformation featuring an isomerized all-trans, ground-state retinal at $115 \mathrm{fs}$. Following Schapiro et al. ${ }^{1}$ these models include two internal water molecules Watı and Wat2 and a solvent-exposed chloride anion. Importantly, these models assume for all titratable residues a specific ionization state, which remains fixed along the computed trajectory. In short, three assumptions were made. ${ }^{25}$ : (i) the protein was placed in vacuum with the two internal water molecules present in their neutral state, and 17 solvent-accessible residues located far from the retinal binding pocket (Asp282, Asp331; Glu5, Glu33, Glu196, Glu201, Glu239, Glu332, Glu341; Lys16, Lys66, Lys67, Lys141, Lys231, Lys311, Lys325, Lys339) were neutralized; (ii) all other residues were assigned a standard protonation state at $\mathrm{pH} 7$ (deprotonated acids, protonated bases) when a counter-ion was present in the vicinity; all His residues except His195 were considered neutral with a single proton placed on $\mathrm{N} \varepsilon 2$; (iii) several internal residues located near the retinal were assigned a neutral state (protonated Asp83, Glu122, Glu181) based on their local environment as well as experimental and/or theoretical evidence obtained from the literature. Here, only the first assumption was retained. A total of 43 residues were considered as titratable (Figure $\mathrm{S}_{4}$ ) among the 65 that the protein contains; they exclude all Ser and Thr residues (see section $\mathrm{S}_{4} \cdot 3$ ), four solvent-exposed His residues (His65, His1oo, His152, His278), and the protonated Schiff Base function.

A consistent set of atomic charges describing either the neutral or charged state of a titratable site must be used for all ionizable residues of the protein. The set of charges to describe most of the protein ionizable residues (Asp, Arg, Glu, His, Lys, Tyr, and the backbone carboxy terminus) was obtained from the $\mathrm{CHARMM}_{3} 6$ force field ${ }^{25,27}$. No parameters were available to describe the ionized states of Ser and Thr; these residues were thus treated as non titratable. The atomic charges for the non-titrating residues were taken from the AMBER force field ${ }^{2}$, i.e., the static electric field produced by the background charges of the protein (section $S_{4.1}$ ) on the retinal along the isomerization path (section $S_{1}$ and $S_{3}$ ) remains the same for all present $\mathrm{pK}_{\mathrm{a}}$ calculations.

The initial PQR structures were post-processed by $\mathrm{CHARMM}^{28}$ to assign a consistent set of charges to all titratable residues for a reference ionization state where all titratable acids (Asp, Glu) and bases (Arg, Lys, His, Tyr) are deprotonated and protonated, respectively. The reference ionization state is needed to build the position of the explicit hydrogens describing the protonated state for bases. For acids, the carboxyl group is described using an implicit hydrogen representation through charge smearing, which lowers the combinatorial complexity; thus the COOH hydrogen was removed from the initially protonated Glu and Asp residues. Missing hydrogen positions for the bases were constructed using the standard HBUILD procedure ${ }^{29}$ in CHARMM followed by a short energy minimization using 100 steps of the Adopted Basis Newton-Raphson (ABNR) algorithm. 
A

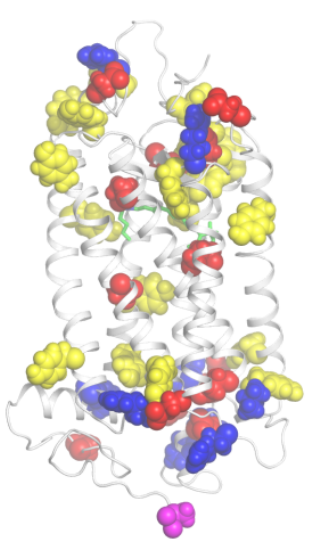

B

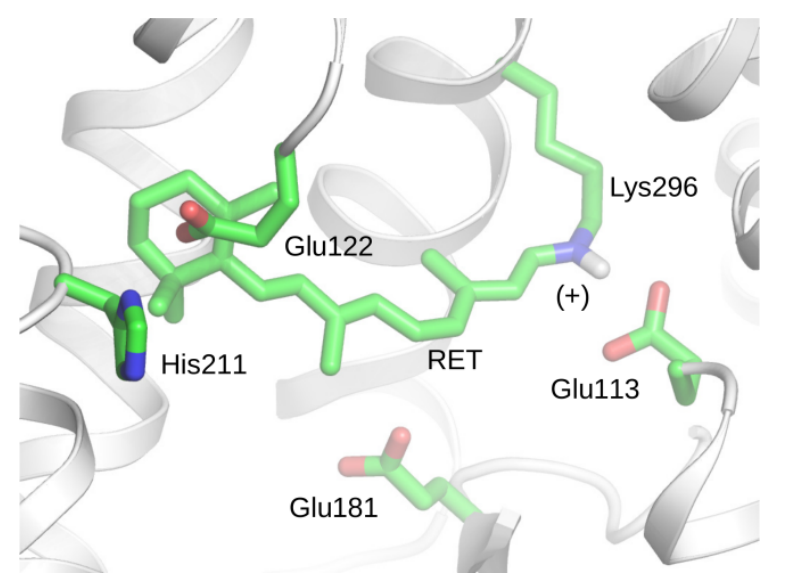

Figure S4. A. Cartoon representation of $\mathrm{Rh}$ with the 43 selected titratable residues highlighted with colored spheres: 3 Asp and 10 Glu (red), 7 Arg and 2 Lys (blue), 18 Tyr and 2 His (yellow), carboxy terminal (magenta). The protein is oriented roughly as in Figure S1. B. close-up view (upside-down) of the retinal binding pocket with the side chains of selected residues and the retinal (RET) shown as colored sticks. Hydrogens atoms are hidden for clarity, except at the protonated Schiff base (PSB) function, which is not titrated in this study. Glu122 and Glu181 were set protonated in the computation of the retinal isomerization path; Glunı is the PSB counter ion.

\section{S4.3 Solvation free energies calculations}

The protein interior was described using a dielectric constant of 4 . The solvent was approximated by a continuum with dielectric constant of 80 to describe bulk water. No membrane model was included in this study as the protein was modeled in vacuum for the calculation of the photoisomerization path (see section $\mathrm{S}_{1}$ ). Including a minimum membrane model in the form of an infinite bi-dimensional low-dielectric slab embedding the protein had no effect on the $\mathrm{pK}_{\mathrm{a}}$ value of the residues in the chromophore cavity. Mobile ions in solution were represented as a Boltzmann-distributed charge density corresponding to a $150-\mathrm{mM}$ solution of $\mathrm{NaCl}$ at $300 \mathrm{~K}$. The thickness of the ion exclusion layer was set to $2.0 \AA$. The solute-solvent dielectric boundary was defined as the solute molecular surface computed with a probe sphere of $1.4 \AA$ radius and atomic radii ${ }^{30}$ of 1.0, 1.50, 1.55, 1.70, and 1.80 $\AA$ for $\mathrm{H}, \mathrm{O}, \mathrm{C}, \mathrm{N}$, and $\mathrm{S}$ atoms, respectively. Using unified atomic radii is important in particular since we are intermixing AMBER and CHARMM force fields in describing the fixed charges and titratable residues (section $\mathrm{S}_{4.2}$ ).

Continuum electrostatic calculations were performed by solving the linearized Poisson-Boltzmann equation over a $3 \mathrm{D}$ grid with the Adaptive Poisson-Boltzmann Solver APBS (41) similar to what was done above in section S3.1. To help convergence, a three-step focusing technique ${ }^{31}$ was used to achieve a final grid spacing of $0.25 \AA$. The tAPBS front-end (http://agknapp.chemie.fu-berlin.de/karlsberg) was employed to ease the calculations required for all titratable sites and collect the corresponding intrinsic $\mathrm{pK}_{\mathrm{a}}$ values and site-site interaction energies (see section $\mathrm{S}_{4.1)}$ ).

\section{S4.4 Titration profiles}

The protonation probability of the titratable groups were obtained by Monte Carlo (MC) optimization, as implemented in the Karlsberg2 program ${ }^{32,33}$. $\mathrm{pK}_{\mathrm{a}}$ values, i.e., the $\mathrm{pH}$ corresponding to the half-protonation point, were derived from onedimensional titration curves for each titratable site calculated by sampling the protonation probability at room temperature over a $\mathrm{pH}$ range from -14 to +28 by increments of $0.1 \mathrm{pH}$ units; see Figure $\mathrm{S}_{5}$ for representative residues. Such a large $\mathrm{pH}$ range was sampled so as to maximize the probability of titrating very acidic or very basic residues (often buried in the protein interior) and derive the corresponding $\mathrm{pK}_{\mathrm{a}}$ value (see Table $\mathrm{S}_{3}$ ). All titratable sites described in section $\mathrm{S}_{4.2}$ were considered. For Histidine residues, the three tautomeric forms, i.e., the imidazole $\mathrm{N} \delta 1$ and $\mathrm{N} \varepsilon 2$ neutral 
forms and the imidazolium charged form, were treated explicitly; here only the pKa corresponding to neutral (single proton) to charged state (doubly-protonated) is reported.

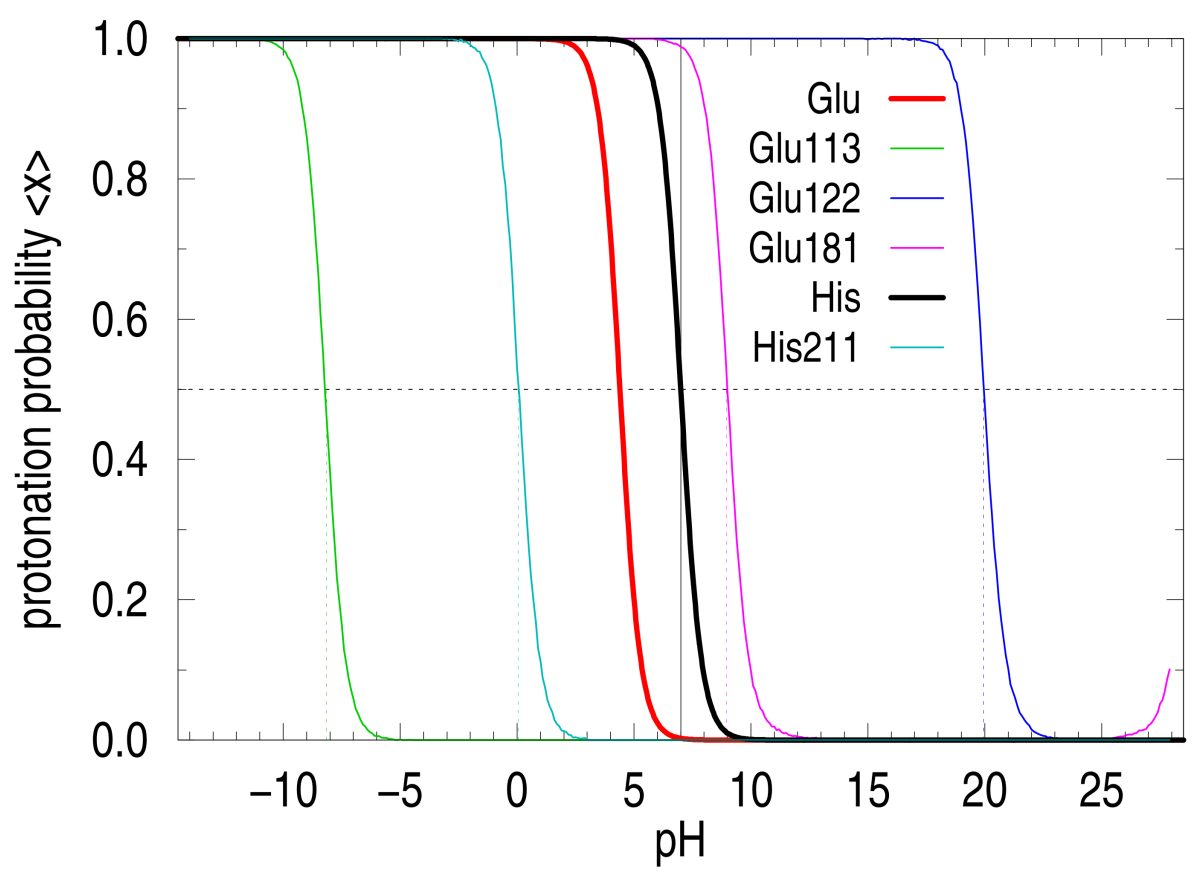

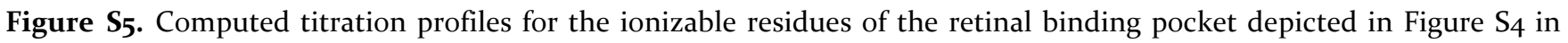
ground-state $\mathrm{Rh}$. The dotted horizontal line shows a probability of 0.5 from which the individual $\mathrm{pK}_{\mathrm{a}}$ values of the protein residues are extracted (colored vertical dotted lines). The vertical solid line emphasizes $\mathrm{pH}=7$. The numberless Glu (in red) and His (in black) traces are the theoretical profiles for the isolated residues in solution (pKa of 4.4 and 7.0, respectively). Glu113 is the PSB counter-ion and is predicted to be very acidic (effective $\mathrm{pK}_{\mathrm{a}}$ of -8.2) due to the facing positive charge of the PSB, which belongs to the non-titrating background charges of the protein. In contrast, Glu122 (blue trace) is predicted to be very basic (predicted $\mathrm{pKa}=20$ ) and thus protonated at $\mathrm{pH}=7$, while His211 is predicted to be much more acidic $(\mathrm{pKa}=0.1)$ and therefore neutral at $\mathrm{pH}=7$ with the proton on $\mathrm{N} \varepsilon 2$. His211 is likely stabilizing the protonated Glu122 via hydrogen bonding with $\mathrm{N} \delta 1$. Glu181 is predicted to be protonated at $\mathrm{pH}=7\left(\mathrm{pK}_{\mathrm{a}}=9\right)$, see Main Text for a discussion on its significance.

Table S3. $\mathrm{pK}_{\mathrm{a}}$ values for the 43 titratable sites sorted by residue type. Std is the standard $\mathrm{pK}_{\mathrm{a}}$ value. Rh configurations are: (\#0) ground-state, (\#1) excited-state, (\#92) near the C.I., (\#115) back to the ground-state along the isomerization path. The Cte348 residue corresponds to the carboxy-terminal titratable group. 


\begin{tabular}{|c|c|c|c|c|c|c|c|c|c|c|c|}
\hline \multirow{2}{*}{ Residue } & \multirow{2}{*}{ Std } & \multicolumn{4}{|c|}{ Configuration \# } & \multirow{2}{*}{ Residue } & \multirow{2}{*}{ Std } & \multicolumn{4}{|c|}{ Configuration \# } \\
\hline & & 0 & 1 & 92 & 115 & & & 0 & 1 & 92 & 115 \\
\hline Arg21 & 12 & 12.7 & 12.7 & 12.7 & 12.7 & His211 & 7 & 0.1 & -0.5 & -1.1 & 0 \\
\hline Arg69 & 12 & 16.5 & 16.5 & 16.5 & 16.4 & Lys245 & 10.4 & 3 & 3 & 3.1 & 3 \\
\hline Arg135 & 12 & 22 & 22 & 21.9 & 22 & Lys 248 & 10.4 & 9.5 & 9.5 & 9.5 & 9.5 \\
\hline Arg147 & 12 & 13.6 & 13.6 & 13.6 & 13.5 & Tyr10 & 9.6 & 12.9 & 12.9 & 12.9 & 12.9 \\
\hline Arg177 & 12 & 16.8 & 16.8 & 16.8 & 16.8 & Tyr29 & 9.6 & 12.8 & 12.8 & 12.9 & 12.8 \\
\hline Arg252 & 12 & 12.7 & 12.7 & 12.7 & 12.7 & Tyr30 & 9.6 & 21.7 & 21.7 & 21.8 & 21.7 \\
\hline Arg314 & 12 & 12.9 & 12.9 & 12.9 & 12.9 & Tyr43 & 9.6 & 15.5 & 16 & 16.1 & 15.4 \\
\hline Asp83 & 4 & 25.1 & 25.3 & 25.3 & 24.9 & Tyr60 & 9.6 & 10 & 10 & 10 & 10 \\
\hline Asp190 & 4 & -3.6 & -4.1 & -4.4 & -3.6 & Tyr74 & 9.6 & 13.5 & 13.5 & 13.5 & 13.5 \\
\hline Asp330 & 4 & 3.3 & 3.3 & 3.3 & 3.3 & Tyr96 & 9.6 & 10.6 & 10.7 & 10.7 & 10.6 \\
\hline Cte348 & 4 & 3.9 & 3.9 & 3.9 & 3.9 & Tyr102 & 9.6 & 12.5 & 12.6 & 12.6 & 12.5 \\
\hline Glu25 & 4.4 & 4 & 4 & 4 & 4 & Tyr136 & 9.6 & 10.7 & 10.7 & 10.7 & 10.7 \\
\hline Glu113 & 4.4 & -8.2 & -3.9 & -0.1 & -7.3 & Tyr178 & 9.6 & 25.6 & 24.7 & 25 & 25.9 \\
\hline Glu122 & 4.4 & 20 & 18 & 16.7 & 19.8 & Tyr191 & 9.6 & $>27.9$ & $>27.9$ & $>27.9$ & $>27.9$ \\
\hline Glu134 & 4.4 & 3.7 & 3.7 & 3.6 & 3.7 & Tyr192 & 9.6 & $>27.9$ & 27.5 & 26.3 & $>27.9$ \\
\hline Glu150 & 4.4 & 3.1 & 3.1 & 3.1 & 3.1 & Tyr206 & 9.6 & 14.7 & 14.5 & 14.7 & 14.7 \\
\hline Glu181 & 4.4 & 9 & 9 & 9.6 & 9.1 & Tyr223 & 9.6 & 11.5 & 11.5 & 11.5 & 11.5 \\
\hline Glu197 & 4.4 & 3.3 & 3.3 & 3.3 & 3.3 & Tyr268 & 9.6 & $>27.9$ & 27.5 & 26.3 & $>27.9$ \\
\hline Glu232 & 4.4 & 4.3 & 4.4 & 4.3 & 4.3 & Tyr274 & 9.6 & 11.2 & 11.2 & 11.2 & 11.2 \\
\hline Glu247 & 4.4 & 0.7 & 0.7 & 0.7 & 0.7 & Tyr301 & 9.6 & 14.9 & 14.7 & 14.9 & 14.8 \\
\hline Glu249 & 4.4 & 5.7 & 5.7 & 5.7 & 5.7 & Tyr306 & 9.6 & 21.3 & 21.3 & 21.3 & 21.3 \\
\hline His195 & 7 & 6.3 & 6.3 & 6.3 & 6.3 & & & & & & \\
\hline
\end{tabular}

\section{References}

(1) Schapiro, I.; Ryazantsev, M. N.; Frutos, L. M.; Ferré, N.; Lindh, R.; Olivucci, M. J. Am. Chem. Soc. 2011, 133, 3354-64.

(2) Cornell, W. D.; Cieplak, P.; Bayly, C. I.; Gould, I. R.; Merz, K. M.; Ferguson, D. M.; Spellmeyer, D. C.; Fox, T.; Caldwell, J. W.; Kollman, P. A. J. Am. Chem. Soc. 1995, 117, 5179-97.

(3) Bashford, D.; Karplus, M. J. Phys. Chem. 1991, 95, 9556-61.

(4) Zheng, G.; Schaefer, M.; Karplus, M. Biochemistry. 2013, 52, 8539-55.

(5) Gozem, S.; Schapiro, I.; Ferre, N.; Olivucci, M. Science. 2012, 337, 1225-8.

(6) Singh, U. C.; Kollman, P. A. J. Comput. Chem. 1986, 7, 718-30.

(7) Roos, B. O.; Taylor, P. R.; Siegbahn, P. E. M. Chemical. Physics. 1980, 48, 157-73.

(8) Andersson, K.; Malmqvist, P. -; Roos, B. O.; Sadlej, A. J.; Wolinski, K. J. J. Phys. Chem. 1990, 94, 5483-8.

(9) Frutos, L. M.; Andruniow, T.; Santoro, F.; Ferré, N.; Olivucci, M. Proc. Natl. Acad. Sci. U. S. A. 2007, 104, 77649.

(10) Aquilante, F.; De Vico, L.; Ferre, N.; Ghigo, G.; Malmqvist, P. A.; Neogrady, P.; Pedersen, T. B.; Pitonak, M.; Reiher, M.; Roos, B. O.; Serrano-Andres, L.; Urban, M.; Veryazov, V.; Lindh, R. J. Comput. Chem. 2010, 31, 22447.

(11) Ponder, J. W.; Richards, F. M. J. Comput. Chem. 1987, 8, 1016-24.

(12) Gozem, S.; Melaccio, F.; Luk, H. L.; Rinaldi, S.; Olivucci, M. Chemical. Society. Reviews. 2014, 43, 4019-36. 
(13) Frisch, M. J.; Trucks, G. W.; Schlegel, H. B.; Scuseria, G. E.; Robb, M. A.; Cheeseman, J. R.; Montgomery, J. A.; Vreven, T.; Kudin, K. N.; Burant, J. C.; Millam, J. M.; Iyengar, S. S.; Tomasi, J.; Barone, V.; Mennucci, B.; Cossi, M.; Scalmani, G.; Rega, N.; Petersson, G. A.; Nakatsuji, H.; Hada, M.; Ehara, M.; Toyota, K.; Fukuda, R.; Hasegawa, J.; Ishida, M.; Nakajima, T.; Honda, Y.; Kitao, O.; Nakai, H.; Klene, M.; Li, X.; Knox, J. E.; Hratchian, H. P.; Cross, J. B.; Bakken, V.; Adamo, C.; Jaramillo, J.; Gomperts, R.; Stratmann, R. E.; Yazyev, O.; Austin, A. J.; Cammi, R.; Pomelli, C.; Ochterski, J. W.; Ayala, P. Y.; Morokuma, K.; Voth, G. A.; Salvador, P.; Dannenberg, J. J.; Zakrzewski, V. G.; Dapprich, S.; Daniels, A. D.; Strain, M. C.; Farkas, O.; Malick, D. K.; Rabuck, A. D.; Raghavachari, K.; Foresman, J. B.; Ortiz, J. V.; Cui, Q.; Baboul, A. G.; Clifford, S.; Cioslowski, J.; Stefanov, B. B.; Liu, G.; Liashenko, A.; Piskorz, P.; Komaromi, I.; Martin, R. L.; Fox, D. J.; Keith, T.; Laham, A.; Peng, C. Y.; Nanayakkara, A.; Challacombe, M.; Gill, P. M. W.; Johnson, B.; Chen, W.; Wong, M. W.; Gonzalez, C.; Pople, J. A. Gaussian 03, Revision C.02 2003, .

(14) Hase, W. L.; Duchovic, R. J.; Hu, X.; Komornicki, A.; Lim, K. F.; Lu, D. -; Peslherbe, G. H.; Swamy, K. N.; Van de Linde, S. R.; Varandas, A. J.. C.. QCPE. 1996, 16, 43.

(15) Tully, J. C. J. Chem. Phys. 1990, 93, 1061-71.

(16) Gozem, S.; Melaccio, F.; Valentini, A.; Filatov, M.; Huix-Rotllant, M.; Ferré, N.; Frutos, L. M.; Angeli, C.; Krylov, A. I.; Granovsky, A. A.; Lindh, R.; Olivucci, M. J. Chem. Theory. Comput. 2014, 10, 3074-84.

(17) Hammes-Schiffer, S.; Tully, J. C. J. Chem. Phys. 1994, 101, 4657-67.

(18) Granucci, G.; Persico, M. J. Chem. Phys. 2007, 126, -.

(19) Humphrey, W.; Dalke, A.; Schulten, K. J. Mol. Graphics. 1996, 14, 33-8.

(20) Baker, N. A.; Sept, D.; Joseph, S.; Holst, M. J.; McCammon, J. A. Proc. Natl. Acad. Sci. U. S. A. 2001, 98, 10037-41.

(21) Dolinsky, T. J.; Nielsen, J. E.; McCammon, J. A.; Baker, N. A. Nucleic. Acids. Res. 2004, 32, W665-7.

(22) Ferré, N.; Cembran, A.; Garavelli, M.; Olivucci, M. Theor. Chem. Acc. 2004, 112, 335-41.

(23) Ullmann, G. M.; Knapp, E. W. Eur. Biophys. J. 1999, 28, 533-51.

(24) Bashford, D.; Karplus, M. Biochemistry. 1990, 29, 10219-25.

(25) Ullmann, G. M.; Knapp, E. W. Eur. Biophys. J. 1999, 28, 533-51.

(26) Calimet, N.; Ullmann, G. M. J. Mol. Biol. 2004, 339, 571-89.

(27) Best, R. B.; Zhu, X.; Shim, J.; Lopes, P. E.; Mittal, J.; Feig, M.; Mackerell, A. D. J. J. Chem. Theory. Comput. 2012, 8, 3257-73.

(28) Brooks, B. R.; Brooks, C. L. 3.; Mackerell, A. D. J.; Nilsson, L.; Petrella, R. J.; Roux, B.; Won, Y.; Archontis, G.; Bartels, C.; Boresch, S.; Caflisch, A.; Caves, L.; Cui, Q.; Dinner, A. R.; Feig, M.; Fischer, S.; Gao, J.; Hodoscek, M.; Im, W.; Kuczera, K.; Lazaridis, T.; Ma, J.; Ovchinnikov, V.; Paci, E.; Pastor, R. W.; Post, C. B.; Pu, J. Z.; Schaefer, M.; Tidor, B.; Venable, R. M.; Woodcock, H. L.; Wu, X.; Yang, W.; York, D. M.; Karplus, M. J. Comput. Chem. 2009, 30, 1545-614.

(29) Brunger, A. T.; Karplus, M. Proteins. 1988, 4, 148-56. 
(30) Bondi, A. J. Phys. Chem. 1964, 68, 441-51.

(31) Klapper, I.; Hagstrom, R.; Fine, R.; Sharp, K.; Honig, B. Proteins. 1986, 1, 47-59.

(32) Rabenstein, B.; Knapp, E. W. Biophys. J. 2001, 80, 1141-50.

(33) Kieseritzky, G.; Knapp, E. W. Proteins. 2008, 71, 1335-48. 